\title{
Symbol-level Precoding for the Non-linear Multiuser MISO Downlink Channel
}

\author{
Danilo Spano, Student Member, IEEE, Maha Alodeh, Member, IEEE, Symeon Chatzinotas, Senior Member, IEEE, \\ Björn Ottersten, Fellow Member, IEEE
}

\begin{abstract}
This paper investigates the problem of the interference among multiple simultaneous transmissions in the downlink channel of a multi-antenna wireless system. A symbol-level precoding scheme is considered, in order to exploit the multiuser interference and transform it into useful power at the receiver side, through a joint utilization of the data information and the channel state information. In this context, this paper presents novel strategies which exploit the potential of symbollevel precoding to control the per-antenna instantaneous transmit power. In particular, the power peaks amongst the transmitting antennas and the instantaneous power imbalances across the different transmitted streams are minimized. These objectives are particularly relevant with respect to the non-linear amplitude and phase distortions induced by the per-antenna amplifiers, which are important sources of performance degradation in practical systems. More specifically, this work proposes two different symbol-level precoding approaches. A first approach performs a weighted per-antenna power minimization, under Qualityof-Service constraints and under a lower bound constraint on the per-antenna transmit power. A second strategy performs a minimization of the spatial peak-to-average power ratio, evaluated amongst the transmitting antennas. Numerical results are presented in a comparative fashion to show the effectiveness of the proposed techniques, which outperform the state of the art symbol-level precoding schemes in terms of spatial peak-toaverage power ratio, spatial dynamic range, and symbol-errorrate over non-linear channels.
\end{abstract}

\section{INTRODUCTION}

A major challenge for next generation wireless communication systems is the increasing demand for higher capacity, to be provided utilizing the available wireless spectrum, which is a scarce resource becoming more and more congested. A possible solution relies on the use of multi-antenna transmitters, which allow aggressive reuse of the frequency spectrum by exploiting the additional degree of freedom given by the spatial dimension. This way, different users can be served by the transmitter sharing the same time and frequency resources, through a space division multiple access scheme [1]. However, full frequency reuse architectures have a crucial limitation

Danilo Spano, Maha Alodeh, Symeon Chatzinotas and Björn Ottersten are with Interdisciplinary Centre for Security Reliability and Trust (SnT) at the University of Luxembourg, Luxembourg. Emails:\{danilo.spano@uni.lu, maha.alodeh@uni.lu, symeon.chatzinotas $@$ uni.lu, and bjorn.ottersten@uni.lu\}.

This work is supported by H2020 project SANSA (Shared Access TerrestrialSatellite Backhaul Network enabled by Smart Antennas), FNR projects SeMiGod (Spectrum Management and Interference Mitigation in Cognitive Radio Satellite Networks) and SATSENT (SATellite SEnsor NeTworks for spectrum monitoring), FNR-EPSRC project CI-PHY (Exploiting interference for physical layer security in 5G networks), and FNR-AFR project BroadSat. Part of this work has been accepted in SPAWC 2017. in the interference between the simultaneous transmissions towards the different co-channel users. As a consequence, advanced signal processing techniques are required to handle the multi-user interference (MUI), in order to boost the overall performance of full frequency reuse systems.

Linear precoding schemes have been shown to be an effective way to manage the MUI, while guaranteeing specific system performance requirements. Precoding techniques can be classified in block-level and symbol-level. In the blocklevel precoding, the transmitted signal is precoded in order to mitigate the MUI using the knowledge of the channel state information (CSI). In this class of techniques, the generic scheme relies on the design of a precoding weight matrix (or precoder), which depends only on the CSI. As a consequence, the precoder remains constant for a whole block of symbols whose length is related to the coherence time of the channel. In this framework, several strategies have been considered for the precoder design [2]-[6], including power minimization schemes with Quality-of-Service (QoS) costraints, as well as max-min fair approaches. The latter ones aim at increasing the fairness of the system, by maximizing the minimum signal-tointerference-plus-noise ratio (SINR) across the users.

On the other hand, the transmitted signals in symbol-level precoding are designed based on the knowledge of both the CSI and the data information, constituted by the symbols to be delivered to the users [7]-[13]. In this approach, the aim is not to cancel the interference, but rather to control it so to have a constructive interference effect at each user. In [7] the classification of the interference as constructive or destructive was given, and a selective channel inversion scheme was proposed in order to eliminate the destructive interference. A more advanced symbol-level precoding scheme was proposed in [8], based on the rotation of the destructive interference so to transform it into useful power. Similarly to the channel-level case, also in this approach different optimization strategies have been considered in the literature. In [9] the sum power minimization and the max-min fair problem were solved for M-PSK modulations. Extensions of such works include optimization strategies for multi-level modulations [10]- [11] and more flexible approaches for exploiting the constructive interference [12]. Furthermore, symbol-level precoding has been considered also in relation to physical layer multicasting [12], and taking into account the imperfect knowledge of the CSI [13].

In the context of symbol-level precoding, this work copes with the following problems:

- In real systems it is common that each individual antenna 
has a dedicated amplifier, resulting in a reduced flexibility in the power allocation amongst the different RF chains of the transmitter. This dictates the need to consider power limitations independently for each antenna, hence to move to a per-antenna based framework.

- The power amplifiers usually introduce non-linear effects which can degrade the transmitted waveform [14], [15]. Therefore, good dynamic properties of the per-antenna transmit power are required to limit the distortion effects. For single-user links, predistortion techniques are used to deal with problem [16]. However, their extension to multi-user systems relying on precoding is not straightforward, due to the complex nature of the constellations produced by the precoding operation.

- With respect to the non-linear effects of the per-antenna amplifiers, an additional degradation is the phase distortion which applies independently to the signals transmitted by each antenna and depends on the transmitted instantaneous power. A high variation between the instantaneous power transmitted by different antennas determines different phase shifts in the amplification stages, and this differential effect is a further source of performance degradation.

To tackle the aforementioned problems, this work introduces novel symbol-level precoding schemes for multi-level modulations aimed at exploiting the constructive interference effect and, at the same time, controlling the instantaneous perantenna power levels. It should be mentioned that this is not possible in the conventional block-level approach, where the precoder is designed for an entire codeword, including several symbols, hence the transmitted power can be controlled only in average and not symbol by symbol. As a consequence, the precoded waveforms can demonstrate unsuitable dynamic properties in terms of power peaks [17]. In particular, the main contributions of this paper can be summarized as follows:

- The non-linear effects, and specifically the differential phase shift effect, are introduced and investigated in the context of precoding.

- Two different symbol-level precoding strategies are proposed, with the objective of reducing the power imbalances between the multiple transmit antennas, in order to have improved performance over non-linear channels with respect to the symbol-level schemes of the literature. In both the proposed approaches QoS constraints are considered to guarantee a per-user required SINR.

- The first presented algorithm minimizes the per-antenna transmit power, and imposes a lower bound to the power carried by each transmitted signal. In this approach, the imbalances between the different RF chains are reduced by constraining the per-antenna transmit power within a specific range.

- The second proposed scheme directly tackles a minimization of the spatial peak-to-average power ratio (SPAPR) amongst the multiple transmit antennas. A simplified version of this SPAPR minimization approach has been presented in [18] for a multi-beam satellite scenario, while herein it is addressed in general multiuser MISO framework.

The main novelty of the proposed techniques with respect to the state of the art on symbol-level precoding [7]-[13] is their ability to exploit the potential of the symbol-level design for producing more robust waveforms to the harmful effects of non-linear channels. It should be noted that a symbol-level precoding scheme accounting for the channel non-linearities has already been proposed by the authors in [19], [20]. Nevertheless, the scheme therein proposed performs just a peak power minimization, without imposing any lower bound nor optimizing the SPAPR. On the other hand, the techniques proposed herein directly aim at a reduction of the power imbalances across the different antennas, therefore they are able to tackle the problem of differential phase shift and to achieve enhanced performance. The scheme of [19], [20] is considered as a benchmark in the numerical evaluation of the proposed techniques, in Section V-C, in order to quantify the relative gains.

It should be also remarked that a number of works available in the literature have proposed precoding techniques suitable for non-linear channels, especially in the context of massive MIMO systems [21]-[23]. In particular, these works aim at reducing the peak-to-average power ratio (PAPR) of the transmitted waveforms, or even at designing constant-envelope signals. A fundamental novelty of this work with respect to [21]-[23] lies in the fact that the proposed techniques are able to leverage the constructive interference effect, while at the same time addressing the problem of non-linearities. As a consequence, the exploitation of the MUI as a beneficial factor constitutes an inherent advantage of the proposed schemes. Another relevant difference is that the techniques proposed herein focus solely on a reduction of the power imbalances in the spatial dimension, i.e., among the different antennas, while the schemes of [21]-[23] allow to improve the dynamic properties of the signals (e.g. the PAPR) also in the temporal dimension. This is due to the fact that the symbol-level precoding formulation with QoS constraints, which is considered in this work, performs the optimization only in a spatial sense, without a direct control of the power variation in the temporal dimension. Overall, the benefit of the techniques proposed herein in terms of constructive interference is counterbalanced by their lack of control of the power dynamic in the temporal dimension. A performance comparison between the proposed schemes and the approach of [21], which designs a constant envelope precoder, is discussed in Section V-C.

Finally, it should be highlighted that this work relies on the assumption of a frequency flat block fading channel. In fact, the proposed precoding techniques are not designed to handle frequency selective channels, as they do not optimize the waveforms in the temporal dimension. This does not allow them to compensate for the inter-symbol interference that arises in frequency selective scenarios. Although a first step towards a spatio-temporal generalization of symbol-level precoding (for linear channels) has been carried out by the authors, and presented in [24], this extension is out of the scope of the present contribution, which is focused on nonlinear systems.

The remainder of the paper is organized as follows. In 
Section II, the system and signals communication model is delineated. In Section III, the problem of weighted per-antenna power minimization with lower bound constraints is proposed and solved. In Section IV, the problem of SPAPR reduction is formulated and solved. In Section V the proposed approach is validated through simulation results. Finally, in Section VI conclusions are drawn.

Notation: We use upper-case and lower-case bold-faced letters to denote matrices and vectors, respectively. $(\cdot)^{T}$ denotes the transpose of $(\cdot)$, while and $(\cdot)^{*}$ and $(\cdot)^{\dagger}$ denote the conjugate and the conjugate transpose of $(\cdot)$, respectively. $|\cdot|$ and $\angle(\cdot)$ denote the amplitude and the phase of $(\cdot)$, respectively, while $\operatorname{Re}(\cdot)$ and $\operatorname{Im}(\cdot)$ are the real and imaginary parts of $(\cdot)$, and $\iota$ is used to denote the imaginary unit. $\|\cdot\|$ and $\|\cdot\|_{\infty}$ represent the Euclidean norm and the $1_{\infty}$ norm of $(\cdot)$, respectively. Moreover, $\operatorname{diag}(\cdot)$ denotes a diagonal matrix whose diagonal entries are the elements of $(\cdot)$, while $\circ$ is used for denoting the element-wise Hadamard operations. $\unrhd$ is used as a generalized inequality for the optimization constraints, to be read as $\geq$ or as = depending whether the constraint is referred to a boundary symbol or to an internal symbol of the constellation, respectively. Finally, $\frac{\partial}{\partial \boldsymbol{x}}$ and $\frac{\partial}{\partial \boldsymbol{x}^{*}}$ denote the gradient operator with respect to $\boldsymbol{x}$ and $\boldsymbol{x}^{*}$, respectively.

\section{System And Signals Model}

We consider a single-cell multiple-antenna downlink scenario, where a single base-station is equipped with $N_{t}$ transmit antennas serving $K$ user terminals, with $N_{t} \geq K$, each one equipped with a single receiving antenna. We assume a block fading channel $\boldsymbol{h}_{j} \in \mathbb{C}^{1 \times N_{t}}$ between the transmit base-station antennas and the $j$-th user. The received signal at the $j$-th user in the symbol slot $n$ can be written as:

$$
y_{j}[n]=\boldsymbol{h}_{j} \boldsymbol{x}[n]+z_{j}[n],
$$

where $\boldsymbol{x}[n] \in \mathbb{C}^{N_{t} \times 1}$ represents the transmitted signal vector from the $N_{t}$ transmit antennas, and $z_{j}[n]$ is a random variable distributed as $\mathcal{C N}\left(0, \sigma_{z}^{2}\right)$, modeling the zero mean Additive White Gaussian Noise (AWGN) measured at the $j$-th user's receiving antenna.

By collecting the received signals by all the users in a vector $\boldsymbol{y}[n] \in \mathbb{C}^{K \times 1}$, the above model can be rewritten in a compact form as:

$$
\boldsymbol{y}[n]=\boldsymbol{H} \boldsymbol{x}[n]+\boldsymbol{z}[n],
$$

where $\boldsymbol{H}=\left[\boldsymbol{h}_{1}^{T} \ldots \boldsymbol{h}_{K}^{T}\right]^{T} \in \mathbb{C}^{K \times N_{t}}$ represents the system channel matrix, and $\boldsymbol{z}[n] \in \mathbb{C}^{K \times 1}$ collects the AWGN components for all the users.

The transmitted signal vector $\boldsymbol{x}[n]$ is obtained as output of a precoding module, which takes as input the CSI, which is an estimate of $\boldsymbol{H}$, and the data information $\boldsymbol{d}[n] \in \mathbb{C}^{K \times 1}$, namely the data symbols to be conveyed to the users. In the transmission scheme we assume a framing structure including a preamble of pilot symbols. Such pilots are exploited by each user to estimate the related channel vector, and the resulting CSI is fed back to the base-station in order to be available for the precoding operation.
In the conventional block-level precoding schemes, the transmitted vector $\boldsymbol{x}[n]$ can be written as $\sum_{j=1}^{K} \boldsymbol{w}_{j}[n] d_{j}[n]$, where $\boldsymbol{w}_{j} \in \mathbb{C}^{N_{t} \times 1}$ represents the precoding vector for the user $j$. Alternatively, by defining a compact precoding matrix $\boldsymbol{W}=\left[\boldsymbol{w}_{1} \ldots \boldsymbol{w}_{K}\right] \in \mathbb{C}^{N_{t} \times K}$, we can write $\boldsymbol{x}[n]=\boldsymbol{W} \boldsymbol{d}[n]$. The matrix $\boldsymbol{W}$ is designed by the precoding module based on an optimization algorithm depending only on the CSI and not on the data information, and for this reason these conventional schemes are also referred to as channel-level precoding. As a consequence, the optimized precoder $\boldsymbol{W}$ changes only when the CSI changes, staying constant for several symbol slots, and the relation between $\boldsymbol{x}[n]$ and $\boldsymbol{d}[n]$ is linear.

In the proposed symbol-level approach the precoding operation uses both the CSI and the data information in order to design the transmitted vector. Thus, this scheme optimizes directly the vector $\boldsymbol{x}[n]$ for every symbol slot without any intermediate steps (such as the design of $\boldsymbol{W}$ ), aiming at constructively exploit the interference. This implies that the relation between the input symbol vector $\boldsymbol{d}[n]$ and the output vector $\boldsymbol{x}[n]$ cannot be explicitly described as in conventional linear precoding, but lies into the optimization design of the precoding module ${ }^{1}$. The data symbols are assumed to be uncorrelated and taken from a generic multi-level constellation represented by the symbol set $\mathcal{D}$, having unit average power, i.e., $\mathbb{E}_{\mathcal{D}}\left[\left|d_{j}\right|^{2}\right]=1$.

\section{A. Non-linear Amplification Stage}

The system model described in (2) is a linear one. However, as already mentioned, it should be considered that the introduced system model is actually corrupted by the non-linear effects introduced in the per-antenna power amplification stages [14], [15], which affect both the amplitude and the phase of the transmitted waveforms. The input-output characteristics of several typologies of high-power amplifiers (HPAs) are available in the literature [15], [17], [25]-[28], including the amplitude-to-amplitude (AM-AM) and the amplitude-to-phase (AM-PM) effects. Moreover, analytical models are available for describing the distortion effects of the HPAs, such as Saleh model [29] for traveling-wave-tube amplifiers (TWTAs) and a modified version [30] for solid state power amplifiers (SSPAs). To model such non-linear effects, we can write in polar coordinates the input signal to the HPA on the generic $i$-th RF chain of the transmitter $a^{2}$ :

$$
x_{i}=\rho_{i} \exp \left(\iota \theta_{i}\right)
$$

where $\rho_{i}$ and $\theta_{i}$ are the amplitude and the phase of $x_{i}$, respectively. Then, the output signal of the HPA can be written as:

$$
\hat{x}_{i}=f_{A}\left(\rho_{i}\right) \exp \left(\iota f_{P}\left(\rho_{i}\right)\right) \exp \left(\iota \theta_{i}\right)
$$

\footnotetext{
${ }^{1}$ In general, it is still possible to formalize the symbol-level precoding scheme in order to design a precoder $\boldsymbol{W}[n]$, such that $\boldsymbol{x}[n]=\boldsymbol{W}[n] \boldsymbol{d}[n]$. However, unlike the block-level schemes, in this case the precoder itself would depend on the data information, thus such intermediate step would actually add a redundant layer of complexity. Therefore, in this work we choose to perform the direct optimization of $\boldsymbol{x}[n]$.

${ }^{2}$ In order to ease the notation, hereafter the time index $n$ is omitted in formulas.
} 


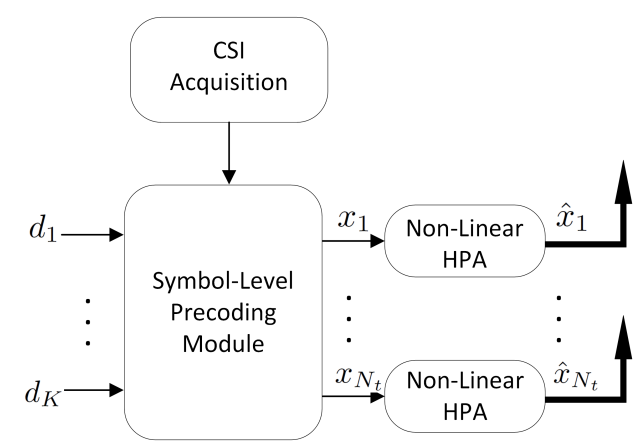

Figure 1: Block scheme of the transmitter relying on symbol-level precoding, for a generic symbol slot.

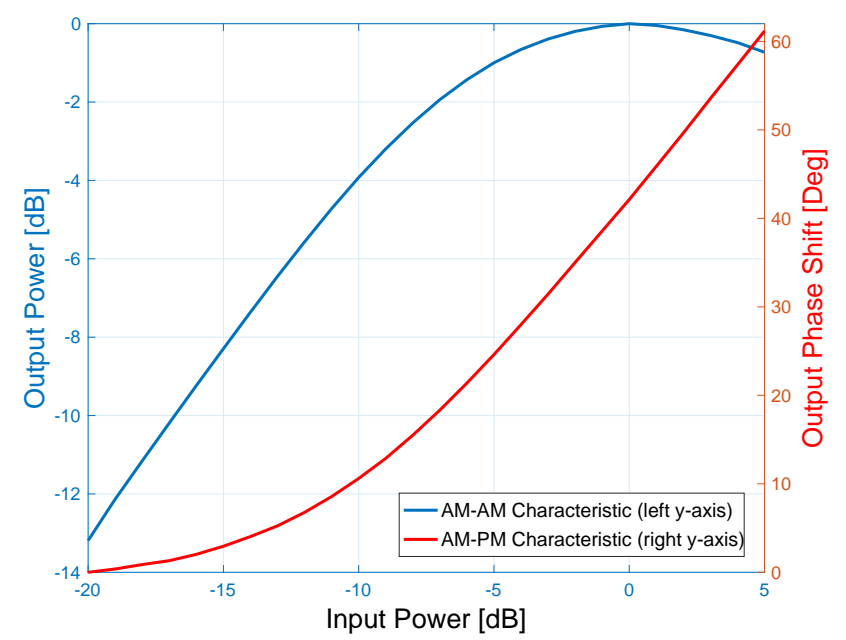

Figure 2: An example of non-linear amplifier: normalized AM-AM and AM-PM characteristics of the non-linearized TWTA of [27].

with $f_{A}(\cdot)$ and $f_{P}(\cdot)$ denoting the AM-AM and the AMPM conversions, respectively. The resulting system model is shown in Fig. 1. A practical example of non-linear HPA is given in Fig. 2, where the (normalized) AM-AM and AM-PM characteristics of the non-linearized TWTA model of [27] are represented. Such characteristics clearly show the saturation effect and the introduced phase distortion, respectively. The model of Fig. 2 is used as a practical reference in this work, in particular for the numerical results section, as it constitutes a highly non-linear model (especially with respect to the AMPM curve) allowing a proper validation of this contribution. However, it should be stressed that all schemes proposed in the remainder of this work are applicable to any non-linear AM-AM/AM-PM characteristic, modeled in the form of (4).

The importance of taking into account the non-linear effects of the amplification stages in the precoding design lies in the two following considerations.

- In some applications, such as satellite communications [17], [26], the power is a scarce resource that has to be efficiently exploited. As a consequence, the HPAs need to be operated as close as possible to their saturation point, and the consequent AM-AM distortion cannot be neglected.
- In other applications the power scarcity is not a crucial issue, therefore stretching the operating region of the HPAs close to saturation is not necessitated. However, there might be the need to employ cheap hardware components for the RF chains, and specifically cheap amplifiers showing more severe non-idealities. This is for example the case of massive-MIMO [31].

In this framework, it is important to control the instantaneous transmitted power and to minimize its peaks, in order to mitigate the performance degradation due to the AM-AM distortion. Furthermore, the use of separate per-antenna HPAs comes with an additional impairment. In fact, as clear from the phase characteristic of the example in Fig. 2, the per-antenna amplifiers introduce a phase shift which is considerably different for different instantaneous powers feeding the HPAs. As a consequence, the precoded data streams transmitted on the separate RF chains will experience a different phase shift through the amplification stages, due to the variable power carried out by the symbols. This specific issue, which will be referred to as differential phase shift, has not been considered in previous literature, and constitutes an additional source of degradation of the overall system performance.

The proposed symbol-level precoding approaches try to cope with the two problems above, namely the saturation effect and the differential phase shift, by reducing the power peaks among the different antennas of the transmitter ${ }^{3}$. In the following sections, these novel precoding strategies are further explained, and the related optimization problems are formalized and solved.

\section{Symbol-Level Precoding for Weighted Peak POWER Minimization With LOWER BOUND (WPPMLB)}

In this section a novel symbol-level precoding scheme is presented, which performs a weighted per-antenna power minimization and imposes a lower bound on the transmit power on each RF chain. According to the general framework of symbol-level precoding, the main objective of the proposed scheme is to design the transmitted vector $\boldsymbol{x}$ by assuring a constructive interference effect at the users' side, following the definition in [9]. In other words, $x$ should be optimized so that the superposition of the multiple streams through the channel forces the received signal to the detection region of the desired symbol, for each user. The novelty of the proposed scheme, with respect to the work on constructive interference carried out in [9], [10], lies in the different optimization of the transmitted power. While the available literature focuses on minimizing the total transmitted power while guaranteeing some QoS targets at the users, here the focus is on the per-antenna transmitted power. Taking into account the non-linear effects of the channel, the goal is to minimize the maximum power among the different antennas and, at the same time, to put a lower bound constraint on such power. This would guarantee a reduction of the power

\footnotetext{
${ }^{3}$ It should be noted that the inter-symbol interference created by the non-linear amplification stages is not modeled in the proposed optimization schemes, as they design the transmit signals only in the spatial dimension, for each symbol slot. However, this effect is taken into account in the numerical evaluation stage of Section V-C.
} 
peaks and a reduced variation of the instantaneous power transmitted by the different antennas, granting better properties with respect to the non-linear amplification stages and, in particular, limiting the differential phase shift. Lastly, the perantenna transmit power is considered in a weighted fashion, so as to account possible asymmetries in the different RF chains. The resulting problem, referred to as weighted peak power minimization with lower bound (WPPMLB), can be written as follows ${ }^{4}$ :

$$
\begin{aligned}
\boldsymbol{x}(\boldsymbol{d}, \boldsymbol{H}, \boldsymbol{\gamma}, \boldsymbol{p})=\arg \min _{r, \boldsymbol{x}} \quad r \\
\text { s.t. } \quad \mathcal{C} 1: \quad \alpha r^{2} \leq \frac{\left|x_{i}\right|^{2}}{p_{i}} \leq r^{2}, i=1, \ldots, N_{t}, \\
\mathcal{C} 2: \quad\left|\boldsymbol{h}_{j} \boldsymbol{x}\right|^{2} \unrhd \kappa_{j}^{2} \gamma_{j} \sigma_{z}^{2}, j=1, \ldots, K, \\
\mathcal{C} 3: \quad \angle \boldsymbol{h}_{j} \boldsymbol{x}=\angle d_{j}, j=1, \ldots, K,
\end{aligned}
$$

where $r$ is a non-negative slack variable used for bounding the power, $\alpha$ is a parameter determining the lower bound (which is better characterized afterwards), $\gamma_{j}$ is the target SINR that should be granted for the $j$-th user, $p_{i}$ is the power weight for the $i$-th antenna, and $\kappa_{j}=\left|d_{j}\right| / \sqrt{\mathbb{E}_{\mathcal{D}}\left[\left|d_{j}\right|^{2}\right]}$ is a magnitude scaling factor for the symbol $d_{j}$, which allows to account the different amplitudes of the symbols in the multilevel constellation $\mathcal{D}$. The assumption to have symbols with unit average power implies that $\kappa_{j}=\left|d_{j}\right|$. Moreover, the vector $\gamma=\left[\gamma_{1} \ldots \gamma_{K}\right]^{T} \in \mathbb{C}^{K \times 1}$ stacks the target SINR for all the users, while $\boldsymbol{p}=\left[p_{1} \ldots p_{N_{t}}\right]^{T} \in \mathbb{C}^{N_{t} \times 1}$ stacks the power weights for all the antennas. Further, the notation $\unrhd$ represents a generalized inequality: it shall be read as $\geq$ or $=$ depending whether the constraint is referred to a boundary symbol or to an inner symbol of the constellation $\mathcal{D}$, respectively (generalized inequalities related to the different detection regions can be also found in [10]).

The set of constraints $\mathcal{C} 1$ in (5) gathers two different kinds of constraints. In particular, such constraints impose an upper bound on the per-antenna weighted transmit power (through the slack variable $r$, so to have a peak power minimization), and a lower bound at the same time. The lower bound is defined through the design parameter $\alpha$. This parameter shall be chosen such that $0 \leq \alpha \leq 1$ and, if considered in $\mathrm{dB}$, represents the width of the region where the transmit power is constrained. The closer to 1 is $\alpha$, the more the power variations will be limited. Nonetheless, the choice of a high value for $\alpha$ comes with a reduction of the degrees of freedom of the optimization problem, whose feasibility is not guaranteed, as discussed afterwards. Concerning the power weights, they are positive parameters affecting the loading of the different RF chains: a higher value for $p_{i}$ implies as higher power loading for the $i$-th antenna with respect to the other ones. The set of constraints $\mathcal{C} 2$ represents a QoS constraint for each user. The set of constraints $\mathcal{C} 3$ represents the constructive interference condition, guaranteeing that each user receives the desired data symbol with the correct phase.

\footnotetext{
${ }^{4}$ It should be mentioned that this formulation of the optimization problem is referred to circular constellations, such as M-PSK and M-APSK. A similar formulation for rectangular constellations, such as M-QAM, can be straightforwardly given but it is not shown for the sake of brevity. This consideration applies to the optimization problems formulated in the remainder of this paper.
}

The problem (5) appears to be complex and hard to tackle. However, it is possible to reformulate it in a more tractable form. More specifically, the following theorem holds, whose proof is reported in the Appendix A:

Theorem 1. The optimization problem (5) is equivalent to the following one:

$$
\begin{aligned}
& \tilde{\boldsymbol{x}}(\boldsymbol{d}, \boldsymbol{H}, \boldsymbol{\gamma}, \boldsymbol{p})= \arg \min _{r, \tilde{\boldsymbol{x}}} \quad r \\
& \text { s.t. } \mathcal{C} 1: \quad \| \boldsymbol{B}_{\boldsymbol{i}} \tilde{\boldsymbol{x}} \leq r, i=1, \ldots, N_{t}, \\
& \mathcal{C} 2: \quad \tilde{\boldsymbol{x}}^{\dagger}\left(\boldsymbol{A}_{i}\right) \tilde{\boldsymbol{x}} \leq-\alpha r^{2}, i=1, \ldots, N_{t}, \\
& \mathcal{C} 3: \quad \operatorname{Re}(\boldsymbol{D}) \boldsymbol{H}_{\mathbf{1}} \tilde{\boldsymbol{x}} \unrhd \boldsymbol{\beta}_{\boldsymbol{R}}, \\
& \mathcal{C} 4: \quad \operatorname{Im}(\boldsymbol{D}) \boldsymbol{H}_{\mathbf{2}} \tilde{\boldsymbol{x}} \unrhd \boldsymbol{\beta}_{\boldsymbol{I}}, \\
& \mathcal{C} 5: \quad\left(\boldsymbol{T} \boldsymbol{H}_{\mathbf{1}}-\boldsymbol{H}_{\mathbf{2}}\right) \tilde{\boldsymbol{x}}=\mathbf{0},
\end{aligned}
$$

where the optimization variable $\tilde{\boldsymbol{x}}$ is a stacked version of $\boldsymbol{x}$, namely $\tilde{\boldsymbol{x}}=\left[\operatorname{Re}(\boldsymbol{x})^{T}, \operatorname{Im}(\boldsymbol{x})^{T}\right]^{T} \in \mathbb{R}^{2 N_{t} \times 1}$, and the other introduced quantities are defined as functions of the input parameters only ${ }^{5}$, with the matrices $\boldsymbol{A}_{i}$ being negative semidefinite $(N S D) \forall i=1, \ldots, N_{t}$.

In particular, the sets of constraints $\mathcal{C} 1$ and $\mathcal{C} 2$ in (6) correspond to the upper and lower bounding of the perantenna transmit power, respectively, while the remaining constraints are related to the attained SINR and the received symbol phases at the different users ${ }^{6}$.

In the problem in (6), it can be seen how all the constraints are convex, with the exception of $\mathcal{C} 2$, imposing the lower bound. Indeed, the upper bound constraint is a second order cone (SOC) constraint, the QoS constraints and the phase constraints are affine, whilst the lower bound constraint is a non-convex, since the matrices $\boldsymbol{A}_{i}$ are NSD.

Hereafter an approach to solve the non-convex problem (6), based on a successive convex approximation (SCA) procedure [32], [33], is proposed.

\section{A. FPP-SCA Algorithm}

The main idea of the SCA algorithm is to iteratively approximate the non-convex problem at hand into a convex one, so to converge to the solution of the original problem. More specifically, assuming a random point $z \in \mathbb{R}^{2 N_{t} \times 1}$, it is always true that $(\tilde{\boldsymbol{x}}-\boldsymbol{z})^{\dagger}\left(\boldsymbol{A}_{i}\right)(\tilde{\boldsymbol{x}}-\boldsymbol{z}) \leq 0$, being $\boldsymbol{A}_{i}$ NSD. Hence, the following inequality holds:

$$
\tilde{\boldsymbol{x}}^{\dagger}\left(\boldsymbol{A}_{i}\right) \tilde{\boldsymbol{x}} \leq 2 \boldsymbol{z}^{\dagger}\left(\boldsymbol{A}_{i}\right) \tilde{\boldsymbol{x}}-\boldsymbol{z}^{\dagger}\left(\boldsymbol{A}_{i}\right) \boldsymbol{z}
$$

which represents a linear restriction of $\tilde{\boldsymbol{x}}^{\dagger}\left(\boldsymbol{A}_{i}\right) \tilde{\boldsymbol{x}}$ around the point $\boldsymbol{z}$. By using the above inequality, the non-convex constraint can be replaced by the following, which is affine in $\tilde{x}$ :

$$
2 \boldsymbol{z}^{\dagger}\left(\boldsymbol{A}_{i}\right) \tilde{\boldsymbol{x}}-\boldsymbol{z}^{\dagger}\left(\boldsymbol{A}_{i}\right) \boldsymbol{z} \leq-\alpha r^{2} .
$$

\footnotetext{
${ }^{5}$ The definition of the introduced vectors and matrices can be found in the proof, and is not reported here for the sake of brevity.

${ }^{6}$ The generalized inequalities $\unrhd$ applied to vectors in the constraints shall be considered element-wise.
} 
By using the above linear restriction, the problem (6) can be written in the following approximated form, to be tackled iteratively:

$$
\begin{aligned}
& \tilde{\boldsymbol{x}}(\boldsymbol{d}, \boldsymbol{H}, \boldsymbol{\gamma}, \boldsymbol{p})=\arg \min _{r, \tilde{\boldsymbol{x}}} r \\
& \text { s.t. } \quad \mathcal{C} 1: \quad\left\|\boldsymbol{B}_{i} \tilde{\boldsymbol{x}}\right\| \leq r, i=1, \ldots, N_{t}, \\
& \mathcal{C} 2: \quad 2 \boldsymbol{z}_{k}^{\dagger}\left(\boldsymbol{A}_{i}\right) \tilde{\boldsymbol{x}}-\boldsymbol{z}_{k}^{\dagger}\left(\boldsymbol{A}_{i}\right) \boldsymbol{z}_{k} \leq-\alpha r^{2}, \\
& i=1, \ldots, N_{t}, \\
& \mathcal{C} 3: \quad \operatorname{Re}(\boldsymbol{D}) \boldsymbol{H}_{\mathbf{1}} \tilde{\boldsymbol{x}} \unrhd \boldsymbol{\beta}_{\boldsymbol{R}}, \\
& \mathcal{C} 4: \quad \operatorname{Im}(\boldsymbol{D}) \boldsymbol{H}_{\mathbf{2}} \tilde{\boldsymbol{x}} \unrhd \boldsymbol{\beta}_{\boldsymbol{I}}, \\
& \mathcal{C} 5:\left(\boldsymbol{T} \boldsymbol{H}_{\mathbf{1}}-\boldsymbol{H}_{\mathbf{2}}\right) \tilde{\boldsymbol{x}}=\mathbf{0},
\end{aligned}
$$

where $\boldsymbol{z}_{k}$ is the introduced auxiliary variable at the $k$-th iteration, which is updated as $\boldsymbol{z}_{k+1}=\tilde{\boldsymbol{x}}$ until convergence, i.e., until $\left\|\tilde{\boldsymbol{x}}-\boldsymbol{z}_{k}\right\|$ is smaller than a predefined threshold.

The introduced approach resorts to the SCA procedure [32][34], which requires the initial point $z_{0}$ to be a feasible one for the original problem. However, since the feasibility of (6) is not guaranteed, it is not easy to find such feasible initial point. To solve the issue, we can rely on the feasible point pursuit SCA (FPP-SCA) algorithm [33]. In particular, the problem can be made always feasible by introducing an additional slack penalty term $s$ as follows:

$$
\begin{aligned}
& \tilde{\boldsymbol{x}}(\boldsymbol{d}, \hat{\boldsymbol{H}}, \boldsymbol{\gamma})= \arg \min _{r, \tilde{\boldsymbol{x}}, \boldsymbol{s}} r+\lambda\|\boldsymbol{s}\| \\
& \text { s.t. } \quad \mathcal{C} 1: \quad\left\|\boldsymbol{B}_{i} \tilde{\boldsymbol{x}}\right\| \leq r+s_{i}, i=1, \ldots, N_{t}, \\
& \mathcal{C} 2: \quad 2 \boldsymbol{z}_{k}^{\dagger}\left(\boldsymbol{A}_{i}\right) \tilde{\boldsymbol{x}}-\boldsymbol{z}_{k}^{\dagger}\left(\boldsymbol{A}_{i}\right) \boldsymbol{z}_{k} \leq-\alpha r^{2}+s_{i+N_{t}}, \\
& i=1, \ldots, N_{t}, \\
& \mathcal{C} 3: \quad \operatorname{Re}(\boldsymbol{D}) \boldsymbol{H}_{\mathbf{1}} \tilde{\boldsymbol{x}} \unrhd \boldsymbol{\beta}_{\boldsymbol{R}}, \\
& \mathcal{C} 4: \quad \operatorname{Im}(\boldsymbol{D}) \boldsymbol{H}_{\mathbf{2}} \tilde{\boldsymbol{x}} \unrhd \boldsymbol{\beta}_{\boldsymbol{I}}, \\
& \mathcal{C} 5: \quad\left(\boldsymbol{T} \boldsymbol{H}_{\mathbf{1}}-\boldsymbol{H}_{\mathbf{2}}\right) \tilde{\boldsymbol{x}}=\mathbf{0},
\end{aligned}
$$

with $s \in \mathbb{R}^{2 N_{t} \times 1}$, and $\lambda$ a trade-off term between the original objective function and the new penalty one. The modified problem in (10) is always feasible for any choice of $\boldsymbol{z}_{0}$ and the convergence is guaranteed [32], [33]. Therefore, the initial point $\boldsymbol{z}_{0}$ can be randomly chosen. If the converged slack penalty variables turn out being all zero, then the related solution solves the original problem (6). In general, the FPPSCA algorithm can be applied by using different starting points $\boldsymbol{z}_{0}$, and then choosing the best solution, namely the one resulting in the lowest-norm penalty term. Concerning the trade-off term $\lambda$, in the fashion of [33] we consider $\lambda \gg 1$, in order to force the penalty terms toward zero, hence pushing the iterates towards the feasible region of the original problem (6), when it is non-empty.

\section{Spatial Peak-to-Average Power Ratio REDUCTION}

In this section, a different symbol-level precoding scheme is proposed, with the objective of directly minimizing the spatial peak-to-average power ratio (SPAPR) amongst the transmit antennas. A metric usually considered in the literature, in the context of non-linear systems, is the peak-to-average power ratio (PAPR) evaluated over time. For example, the temporal PAPR has been tackled for OFDM systems [22], [35], [36]. Here we focus instead on the SPAPR which, in light of the impairments described in Section II-A, is also important in order to utilize the multiple amplifiers in a more homogeneous way. The SPAPR can be defined as:

$$
\operatorname{SPAPR}=\frac{\|\boldsymbol{x}\|_{\infty}^{2}}{\|\boldsymbol{x}\|^{2} / N_{t}} .
$$

In the direction of designing the transmit waveforms controlling their instantaneous power, the opportunity to have low SPAPR allows a reduction of the per-antenna power imbalances across the different HPAs, thus limiting the related differential phase shift.

\section{A. Spatial PAPR Optimization}

The SPAPR minimization (SPAPR-Min) problem can be formulated as non-linear fractional program, as:

$$
\begin{aligned}
\boldsymbol{x}(\boldsymbol{d}, \boldsymbol{H}, \boldsymbol{\gamma})= & \arg \min _{\boldsymbol{x}} \frac{\|\boldsymbol{x}\|_{\infty}^{2}}{\|\boldsymbol{x}\|^{2}} \\
\text { s.t. } & \mathcal{C} 1: \quad\left|\boldsymbol{h}_{j} \boldsymbol{x}\right|^{2} \unrhd \kappa_{j}^{2} \gamma_{j} \sigma_{z}^{2}, j=1, \ldots, K, \\
& \mathcal{C} 2: \quad \angle \boldsymbol{h}_{j} \boldsymbol{x}=\angle d_{j}, j=1, \ldots, K .
\end{aligned}
$$

It is possible to reformulate the problem (12), as shown in the following theorem, whose proof is reported in the Appendix B:

Theorem 2. The optimization problem (12) is equivalent to the following one:

$$
\begin{aligned}
\boldsymbol{x}(\boldsymbol{d}, \boldsymbol{H}, \boldsymbol{\gamma})= & \arg \min _{\boldsymbol{x}} \frac{\|\boldsymbol{x}\|_{\infty}^{2}}{\|\boldsymbol{x}\|^{2}} \\
\text { s.t. } \quad \mathcal{C} 1: \quad & \operatorname{Re}\left(d_{j}\right) \frac{\boldsymbol{h}_{j} \boldsymbol{x}+\boldsymbol{x}^{\dagger} \boldsymbol{h}_{j}^{\dagger}}{2} \unrhd \sigma_{z} \sqrt{\gamma_{j}} \operatorname{Re}^{2}\left(d_{j}\right), \\
& j=1, \ldots, K, \\
\mathcal{C} 2: \quad & \operatorname{Im}\left(d_{j}\right) \frac{\boldsymbol{h}_{j} \boldsymbol{x}-\boldsymbol{x}^{\dagger} \boldsymbol{h}_{j}^{\dagger}}{2 \iota} \unrhd \sigma_{z} \sqrt{\gamma_{j}} \operatorname{Im}^{2}\left(d_{j}\right), \\
& j=1, \ldots, K, \\
\mathcal{C} 3: \quad & t_{j}\left(\iota t_{j}-1\right) \boldsymbol{h}_{j} \boldsymbol{x}+\left(\iota t_{j}+1\right) \boldsymbol{x}^{\dagger} \boldsymbol{h}^{\dagger}=0, \\
& j=1, \ldots, K .
\end{aligned}
$$

where $t_{j}=\tan \left(\angle d_{j}\right)$.

In the reformulated problem (13), the challenging part is in the non-linear fractional objective function. Dinkelbach suggests a parametric way of solving the non-linear fractional problems [37], [38], whose basic idea is to tackle the fractional problem by solving a sequence of easier problems which converges to the global solution. Nevertheless, Dinkelbach's algorithm can be applied only if the numerator and denominator are convex and concave, respectively. Therefore, it cannot be directly applied to the problem at hand, since the numerator 
$\|\boldsymbol{x}\|_{\infty}^{2}$ and denominator $\|\boldsymbol{x}\|^{2}$ are both convex functions. In order to solve the problem, we can again resort to a SCA approach [32]-[34]. In particular, we can approximate the quadratic function around a certain vector $\boldsymbol{z} \in \mathbb{C}^{N_{t} \times 1}$ by a concave (affine) function as:

$$
\begin{aligned}
(\boldsymbol{x}-\boldsymbol{z})^{\dagger}(\boldsymbol{x}-\boldsymbol{z})= & \boldsymbol{x}^{\dagger} \boldsymbol{x}-2 \operatorname{Re}\left(\boldsymbol{z}^{\dagger} \boldsymbol{x}\right)+\boldsymbol{z}^{\dagger} \boldsymbol{z} \geq 0 \\
& \boldsymbol{x}^{\dagger} \boldsymbol{x} \geq 2 \operatorname{Re}\left(\boldsymbol{z}^{\dagger} \boldsymbol{x}\right)-\boldsymbol{z}^{\dagger} \boldsymbol{z} \\
& \boldsymbol{x}^{\dagger} \boldsymbol{x} \approx 2 \operatorname{Re}\left(\boldsymbol{z}^{\dagger} \boldsymbol{x}\right)-\boldsymbol{z}^{\dagger} \boldsymbol{z} .
\end{aligned}
$$

Using this lower bound approximation, the problem in (12) can be rewritten as:

$$
\begin{aligned}
\boldsymbol{x}(\boldsymbol{d}, \boldsymbol{H}, \boldsymbol{\gamma})= & \arg \min _{\boldsymbol{x}} \frac{\|\boldsymbol{x}\|_{\infty}^{2}}{\left(2 \operatorname{Re}\left(\boldsymbol{z}^{\dagger} \boldsymbol{x}\right)-\boldsymbol{z}^{\dagger} \boldsymbol{z}\right)} \\
\text { s.t. } \quad \mathcal{C} 1: \quad & \operatorname{Re}\left(d_{j}\right) \frac{\boldsymbol{h}_{j} \boldsymbol{x}+\boldsymbol{x}^{\dagger} \boldsymbol{h}_{j}^{\dagger}}{2} \unrhd \sigma_{z} \sqrt{\gamma_{j}} \operatorname{Re}^{2}\left(d_{j}\right), \\
& j=1, \ldots, K, \\
\mathcal{C} 2: \quad & \operatorname{Im}\left(d_{j}\right) \frac{\boldsymbol{h}_{j} \boldsymbol{x}-\boldsymbol{x}^{\dagger} \boldsymbol{h}_{j}^{\dagger}}{2 \iota} \unrhd \sigma_{z} \sqrt{\gamma_{j}} \operatorname{Im}^{2}\left(d_{j}\right), \\
& j=1, \ldots, K, \\
\mathcal{C} 3: \quad & t_{j}\left(\iota t_{j}-1\right) \boldsymbol{h}_{j} \boldsymbol{x}+\left(\iota t_{j}+1\right) \boldsymbol{x}^{\dagger} \boldsymbol{h}^{\dagger}=0, \\
& j=1, \ldots, K .
\end{aligned}
$$

Now the problem can be solved by applying parametric programming on the approximated formulation in (15). To this aim, we should define the optimization function $F(\eta)=$ $\min _{\boldsymbol{x} \in \mathcal{S}}\left\{\|\boldsymbol{x}\|_{\infty}^{2}-\eta\left(2 \operatorname{Re}\left(\boldsymbol{z}_{k}^{\dagger} \boldsymbol{x}\right)-\boldsymbol{z}_{k}^{\dagger} \boldsymbol{z}_{k}\right)\right\}$, where $\mathcal{S}$ represents the sets of constraints $\mathcal{C}_{1}, \mathcal{C}_{2}$ and $\eta$ is an auxiliary variable to apply parametric programming techniques. Therefore, the problem can be formulated as:

$$
\begin{aligned}
\boldsymbol{x}(\boldsymbol{d}, \boldsymbol{H}, \boldsymbol{\gamma})= & \arg \min _{\boldsymbol{x}, \eta} \quad\|\boldsymbol{x}\|_{\infty}^{2}-\eta\left(2 \operatorname{Re}\left(\boldsymbol{z}^{\dagger} \boldsymbol{x}\right)-\boldsymbol{z}^{\dagger} \boldsymbol{z}\right) \\
\text { s.t. } \quad \mathcal{C} 1: \quad & \operatorname{Re}\left(d_{j}\right) \frac{\boldsymbol{h}_{j} \boldsymbol{x}+\boldsymbol{x}^{\dagger} \boldsymbol{h}_{j}^{\dagger}}{2} \unrhd \sigma_{z} \sqrt{\gamma_{j}} \operatorname{Re}^{2}\left(d_{j}\right), \\
& j=1, \ldots, K, \\
\mathcal{C} 2: \quad & \operatorname{Im}\left(d_{j}\right) \frac{\boldsymbol{h}_{j} \boldsymbol{x}-\boldsymbol{x}^{\dagger} \boldsymbol{h}_{j}^{\dagger}}{2 \iota} \unrhd \sigma_{z} \sqrt{\gamma_{j}} \operatorname{Im}^{2}\left(d_{j}\right), \\
& j=1, \ldots, K, \\
\mathcal{C} 3: \quad & t_{j}\left(\iota t_{j}-1\right) \boldsymbol{h}_{j} \boldsymbol{x}+\left(\iota t_{j}+1\right) \boldsymbol{x}^{\dagger} \boldsymbol{h}^{\dagger}=0, \\
& j=1, \ldots, K .
\end{aligned}
$$

Ultimately, to efficiently solve (13) using the formulation in (16), we propose the algorithm in Table I. The theoretical lower bound occurs when all the antennas have the same power (i.e., $\|\boldsymbol{x}\|^{2} / N_{t}$ ), with an achieved unit SPAPR.

\section{B. Convergence of the Algorithm}

It is proven in [37] that the parametric programming scheme applied to concave/linear fractional programs converge to a global optimum (if the objective is to maximize the concave/linear function). In our case this holds, since the objective is to minimize a convex/linear function.

On the other hand, considering the SCA approach, it is proven convergent [32] to a Karush-Kuhn-Tucker (KKT) point,
Table I: Proposed Successive Linear Approximation for Non-linear Fractional Programming

1) Initialization: Set $\epsilon, k=0, \eta=0$ in (16), which results in solving $F(0)$.

2) Evaluate $\eta_{0}=\frac{\|\boldsymbol{x}\|_{\infty}^{2}}{\|\boldsymbol{x}\|^{2}}, \boldsymbol{z}_{0}=\boldsymbol{x}$.

3) Solve the following optimization:

$$
\begin{aligned}
& \qquad \boldsymbol{x}=\arg \min _{\boldsymbol{x}}\|\boldsymbol{x}\|_{\infty}^{2}-\eta_{k}\left(2 \operatorname{Re}\left(\boldsymbol{z}_{k}^{\dagger} \boldsymbol{x}\right)-\boldsymbol{z}_{k}^{\dagger} \boldsymbol{z}_{k}\right) \\
& \text { s.t. } \quad \mathcal{C} 1: \quad \operatorname{Re}\left(d_{j}\right) \frac{\boldsymbol{h}_{j} \boldsymbol{x}+\boldsymbol{x}^{\dagger} \boldsymbol{h}_{j}^{\dagger}}{2} \unrhd \sigma_{z} \sqrt{\gamma_{j}} \operatorname{Re}^{2}\left(d_{j}\right), j=1, \ldots, K, \\
& \mathcal{C} 2: \quad \operatorname{Im}\left(d_{j}\right) \frac{\boldsymbol{h}_{j} \boldsymbol{x}-\boldsymbol{x}^{\dagger} \boldsymbol{h}_{j}^{\dagger}}{2 \iota} \unrhd \sigma_{z} \sqrt{\gamma_{j}} \operatorname{Im}^{2}\left(d_{j}\right), j=1, \ldots, K, \\
& \mathcal{C} 3: \quad t_{j}\left(\iota t_{j}-1\right) \boldsymbol{h}_{j} \boldsymbol{x}+\left(\iota t_{j}+1\right) \boldsymbol{x}^{\dagger} \boldsymbol{h}^{\dagger}=0, j=1, \ldots, K . \\
& \text { 4) Evaluate }\left|F\left(\eta_{k}\right)\right| \text { and }\left\|\boldsymbol{x}-\boldsymbol{z}_{k}\right\| ; \text { if }\left|F\left(\eta_{k}\right)\right| \geq \epsilon \text { or }\left\|\boldsymbol{x}-\boldsymbol{z}_{k}\right\| \geq \epsilon \\
& \text { go to step 5. } \\
& \text { 5) Set } \eta_{k+1}=\frac{\|\boldsymbol{x}\|_{\infty}^{2}}{\|\boldsymbol{x}\|^{2}}, \boldsymbol{z}_{k+1}=\boldsymbol{x}, k=k+1, \text { go to step 3. }
\end{aligned}
$$

provided that the approximation is a concave lower bound having the same first order behavior of the original function. This is the case of the approximation in (14), as stated in the following proposition.

Proposition 1. Given the convex function $f(\boldsymbol{x})=\|\boldsymbol{x}\|^{2}$, and its concave (affine) approximation around $\boldsymbol{z} \tilde{f}(\boldsymbol{x}, \boldsymbol{z})=$ $2 \operatorname{Re}\left(\boldsymbol{z}^{\dagger} \boldsymbol{x}\right)-\boldsymbol{z}^{\dagger} \boldsymbol{z}$, the following properties hold:

$$
\begin{aligned}
\tilde{f}(\boldsymbol{x}, \boldsymbol{z}) & \leq f(\boldsymbol{x}), \\
\tilde{f}(\boldsymbol{x}, \boldsymbol{x}) & =f(\boldsymbol{x}), \\
\left.\frac{\partial}{\partial \boldsymbol{x}} \tilde{f}(\boldsymbol{x}, \boldsymbol{z})\right|_{\boldsymbol{z}=\boldsymbol{x}} & =\frac{\partial}{\partial \boldsymbol{x}} f(\boldsymbol{x}), \\
\left.\frac{\partial}{\partial \boldsymbol{x}^{*}} \tilde{f}(\boldsymbol{x}, \boldsymbol{z})\right|_{\boldsymbol{z}=\boldsymbol{x}} & =\frac{\partial}{\partial \boldsymbol{x}^{*}} f(\boldsymbol{x}),
\end{aligned}
$$

where the gradient is considered with respect to $\boldsymbol{x}$ and $\boldsymbol{x}^{*}$, and these variable are treated as independent, in the fashion of [39].

Proof. The properties (18) and (19) come straightforwardly from (14). In order to prove (20) and (21), we derive hereafter the gradient of $f(\boldsymbol{x})$ and $\tilde{f}(\boldsymbol{x}, \boldsymbol{z})$ with respect to $\boldsymbol{x}$ and $\boldsymbol{x}^{*}$, based on [39]:

$$
\begin{aligned}
\frac{\partial}{\partial \boldsymbol{x}} f(\boldsymbol{x}) & =\frac{\partial}{\partial \boldsymbol{x}}\left\{\boldsymbol{x}^{\dagger} \boldsymbol{x}\right\}=\boldsymbol{x}^{\dagger}, \\
\frac{\partial}{\partial \boldsymbol{x}^{*}} f(\boldsymbol{x}) & =\frac{\partial}{\partial \boldsymbol{x}^{*}}\left\{\boldsymbol{x}^{\dagger} \boldsymbol{x}\right\}=\boldsymbol{x}^{T}, \\
\frac{\partial}{\partial \boldsymbol{x}} \tilde{f}(\boldsymbol{x}, \boldsymbol{z}) & =\frac{\partial}{\partial \boldsymbol{x}}\left\{\boldsymbol{z}^{\dagger} \boldsymbol{x}+\boldsymbol{x}^{\dagger} \boldsymbol{z}\right\}=\boldsymbol{z}^{\dagger}, \\
\frac{\partial}{\partial \boldsymbol{x}^{*}} \tilde{f}(\boldsymbol{x}, \boldsymbol{z}) & =\frac{\partial}{\partial \boldsymbol{x}^{*}}\left\{\boldsymbol{z}^{\dagger} \boldsymbol{x}+\boldsymbol{x}^{\dagger} \boldsymbol{z}\right\}=\boldsymbol{z}^{T} .
\end{aligned}
$$

By evaluating (24) and (25) in $\boldsymbol{z}=\boldsymbol{x}$, the properties (20) and (21) follows, and the proposition is proved.

Although the parametric programming and the SCA approaches have been shown convergent individually, it shall be 


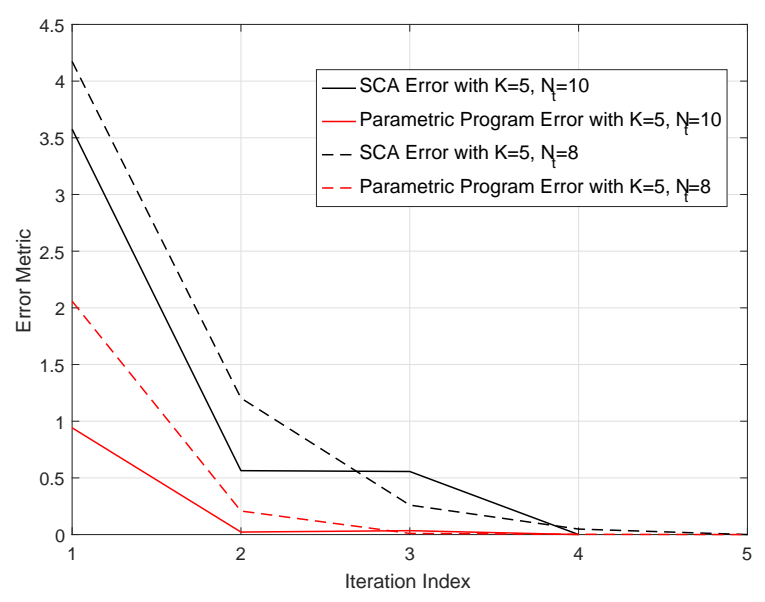

Figure 3: Error metrics of the algorithm of I, versus iteration index, for two instances of the algorithm.

noticed how the formulation (16), and accordingly the algorithm proposed in Table I, employs these schemes in a joint fashion, and it is not straightforward to prove the convergence of the final algorithm. However, the proposed scheme has been shown convergent through numerical simulations. This is shown in Fig. 3 for two instances of the algorithm, with $K=5$ and $N_{t}$ set to 10 and 8, respectively. The figure shows, for both the cases, how the SCA error $\left\|\boldsymbol{x}-\boldsymbol{z}_{k}\right\|$ and the parametric programming error $\left|F\left(\eta_{k}\right)\right|$ vary with respect to the iteration index, going to zero in a few iterations.

\section{Numerical Results}

In this section some numerical results are presented, to show the effectiveness of the proposed approaches, in particular the WPPMLB scheme and the SPAPR-Min sheme. Before discussing the results, let us introduce the considered performance metrics. The most prominent metrics with respect to the problem of non-linearities, which is the main object of this contribution, are the SPAPR and the spatial dynamic range. The former has been already defined in equation (11), while the latter is defined, for a specific symbol slot, as the ratio between the maximum and the minimum transmit power amongst the antennas, i.e., as $\frac{\|\boldsymbol{x}\|_{\infty}^{2}}{\min \left|x_{i}\right|^{2}}$. Moreover, the total transmit power and the average achieved SINR are also considered for the performance evaluation. The introduced quantities, which are symbol dependent by definition, are considered at a frame level by averaging over a large number of symbol slots. An additional performance metric used in this section is the symbol error rate (SER), which is useful to quantify the effectiveness of the proposed techniques when a non-linear channel is applied in the communication chain.

All the results presented in the remainder of this section are obtained assuming a 16-APSK modulation scheme for the data symbols, while the number of users is fixed to $K=5$. The quasi-static block fading channel coefficients have been generated, for the generic user $j$, as $\boldsymbol{h}_{j} \sim \mathcal{C N}\left(0, \sigma_{h}^{2} \boldsymbol{I}\right)$, with $\sigma_{h}^{2}=1$. The results have been obtained averaging over 30 fading blocks of 20 symbol slots each, for a total of
600 realizations. Moreover, the noise variance $\sigma_{z}^{2}$ is assumed unitary. Finally, the target SINR is assumed the same for all the users for the sake of simplicity, and it is fixed to $12 \mathrm{~dB}$ for all the results ${ }^{7}$.

In the following, the performance of the WPPMLB and SPAPR-Min schemes are analyzed with respect to some parameters, such as the number of transmit antennas and the input $\alpha$ (for the WPPMLB case). Then, some comparative results are presented, in order to compare the proposed techniques to the benchmarks, i.e., the sum power minimization approach of [9], [10], the peak power minimization scheme of [19], [20], and the constant envelope precoder of [21].

\section{A. Performance of WPPMLB Scheme with respect to the Parameters}

Hereafter, the performance of the WPPMLB scheme is investigated with respect to the number of transmit antennas and to the value of the design parameter $\alpha$ in the problem (5). Concerning this, it is worth noticing that the value $1 / \alpha$ represents the imposed spatial dynamic range for the transmit signal. Nevertheless, it has been mentioned how the imposition of a tight spatial dynamic range (i.e., $\alpha$ close to 1 ) may compromise the feasibility of the problem, since it implies a reduction in the degrees of freedom. As a consequence, it is imperative to study to which extent it is possible to constraint the WPPMLB problem, and how this is affected by the number of transmit antennas $N_{t}$. The presented results are obtained by running the FPP-SCA algorithm using two random starting points and then choosing the best solution, as discussed in Section III-A. Moreover, the power weights are assumed equal to one, for simplicity.

In Fig. 4 the attained spatial dynamic range is displayed as a function of the imposed one (i.e. $1 / \alpha$ ), in $\mathrm{dB}$, for different values of $N_{t}$. It is apparent how, when the number of transmit antennas is equal to the number of users (fixed to 5), the attained spatial dynamic range is larger than the required one for almost all the simulated values. In other words, in this case the WPPMLB problem (5) turns out not to be feasible for all the symbols and channel realizations ${ }^{8}$, unless the imposed spatial dynamic range is very large (over $7 \mathrm{~dB}$ ). It can be also noticed how, due to the infeasibility of the optimization problem, the attained spatial dynamic range shows even a decreasing trend with $1 / \alpha$ when the latter is below $2 \mathrm{~dB}$. This means that, in the case of 5 antennas, reducing too much the imposed spatial dynamic range can even worsen the performance. On the other hand, by increasing $N_{t}$ the optimization problem is relaxed, and it is possible to respect the imposed constraints also with a reduced spatial dynamic range, as it is visible from the results obtained with $N_{t}=8$ and $N_{t}=10$. Remarkably, in the latter case the problem is feasible even when a unit spatial dynamic range $(0 \mathrm{~dB})$ is imposed. The dependency of the problem feasibility on the imposed spatial dynamic range and on the number of antennas is further

\footnotetext{
${ }^{7}$ This does not apply for the results in Figs. 8, 10, where the power-SINR dependence is studied.

${ }^{8}$ This implies that the lower bound constraints on the power are not met in average, as clear from the displayed result.
} 


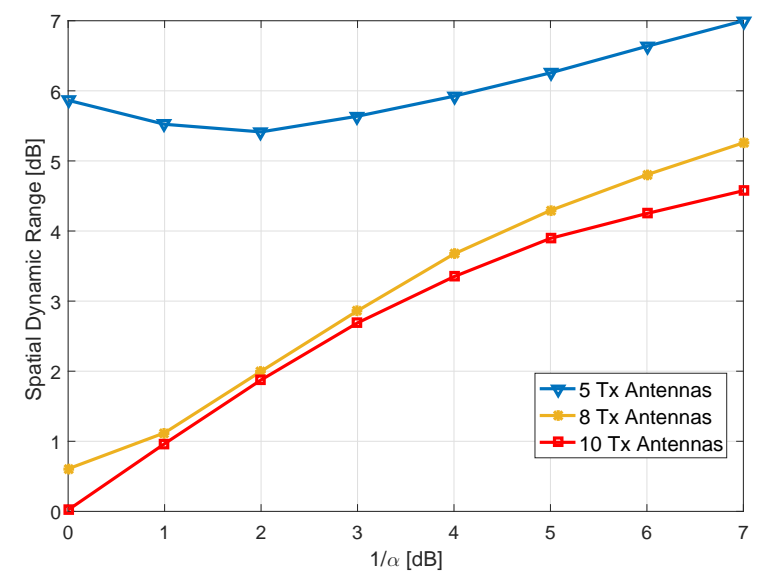

Figure 4: Attained spatial dynamic range, in $\mathrm{dB}$, versus imposed one in $\mathrm{dB}$.

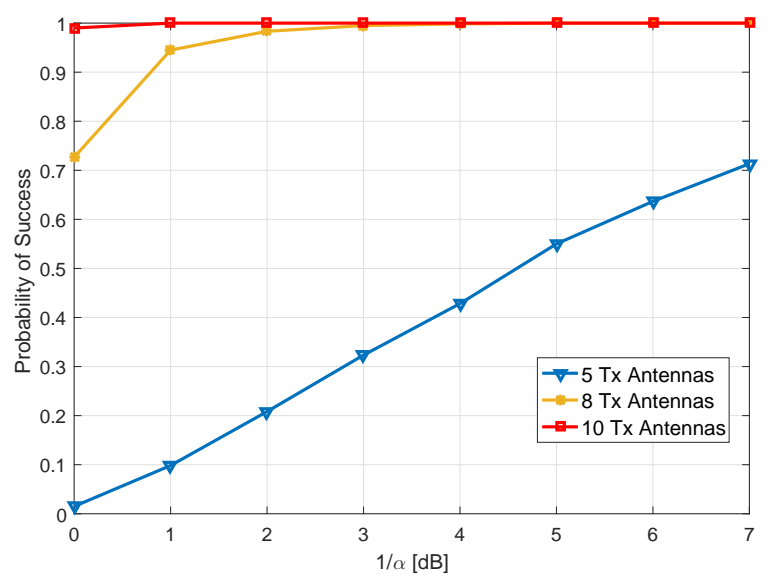

Figure 5: Probability of success versus imposed spatial dynamic range in $\mathrm{dB}$.

shown in Fig. 5, where the probability of success in solving the WPPMLB problem is shown. This quantity, which is the probability of respecting the imposed constraint on the spatial dynamic range, is calculated through a Monte Carlo simulation over the multiple channel and symbols realizations. This figure shows how the probability of success is low for $N_{t}=5$, and how it strongly decreases when $1 / \alpha$ is reduced. This explains the decreasing trend observed in Fig. 4 for $1 / \alpha$ below 2 dB. Increasing the number of transmit antennas the probability of success increases considerably, becoming basically 1 for any imposed spatial dynamic range when $N_{t}=10$.

In Fig. 6 the total transmit power, in dBW, is shown as a function of the imposed spatial dynamic range, for 8 and 10 transmit antennas. The case with only 5 antennas is not considered, since the problem is not feasible for basically all the values of $1 / \alpha$, as previously discussed. It can be seen how the configuration with $\alpha=0$ requires a high transmit power ${ }^{9}$,

\footnotetext{
${ }^{9}$ However, it should be kept in mind that we are assuming a reference scenario with unit noise variance at the receivers' side, so the results in terms of transmit power shall be interpreted more in a comparative fashion than in an absolute way.
}

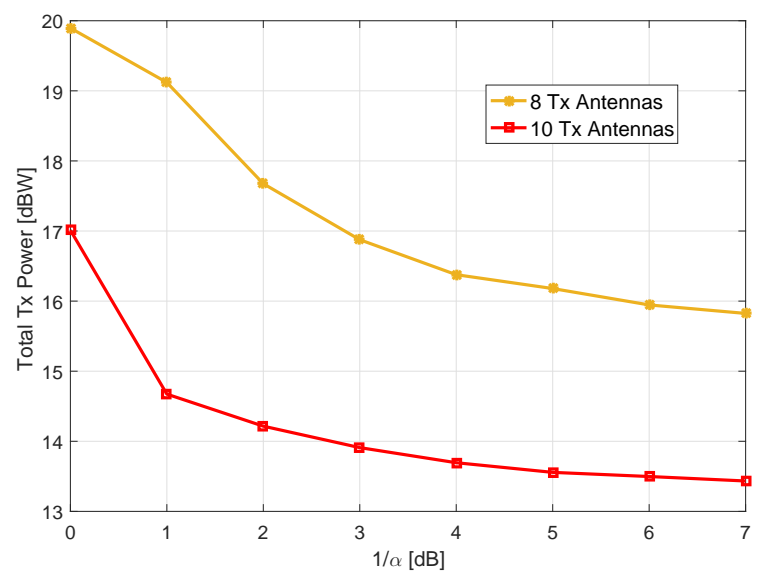

Figure 6: Total transmit power, in $\mathrm{dBW}$, versus imposed spatial dynamic range in $\mathrm{dB}$.

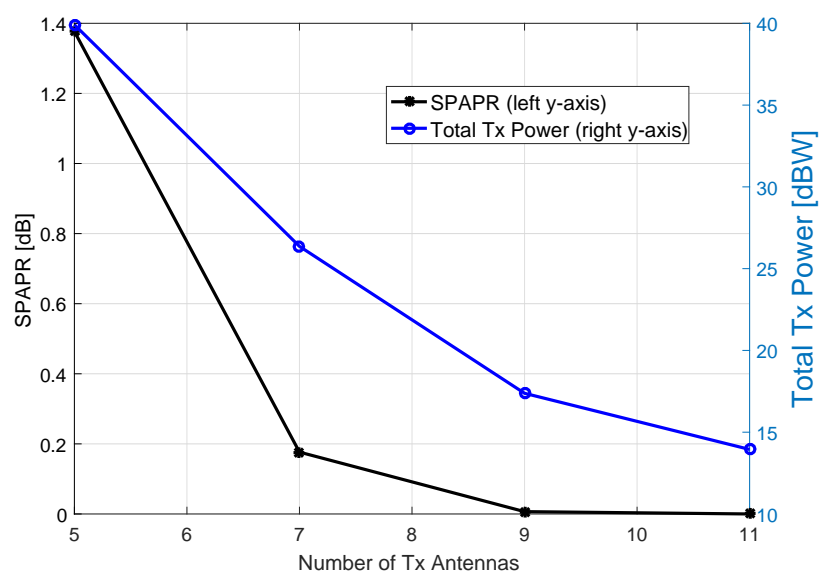

Figure 7: Attained SPAPR, in $\mathrm{dB}$, versus number of transmit antennas.

because of the very tight constraint, especially in the case with 8 antennas. Nevertheless, the result shows how a relaxation of the constraint on the imposed spatial dynamic range, as well as an increase in the number of antennas, allows to reduce the transmit power. Such behavior is due to an increase of the degrees of freedom of the optimization problem when the constraint is relaxed or more antennas are used.

\section{B. Performance of SPAPR-Min Scheme with respect to the Number of Tx Antennas}

Herein we examine how the performance of the SPAPRMin scheme depends on the number of transmit antennas $N_{t}$ considered in the system. This dependency can be seen in Fig. 7, which displays the attained SPAPR and the total transmit power as functions of $N_{t}$. Similarly to the previous problem, also here we can observe how increasing the number of transmit antennas implies better performance. In particular, the attained SPAPR decreases when $N_{t}$ increases, reaching the theoretical lower bound of $0 \mathrm{~dB}$ for $N_{t} \geq 9$. Moreover, the total transmit power sensibly decreases when $N_{t}$ increases. 
Table II: Summary of the considered symbol-level precoding techniques.

\begin{tabular}{llc}
\hline Technique Acronym & Extended Name & Problem Ref. \\
\hline WPPMLB & $\begin{array}{l}\text { Weighted Peak Power Mini- } \\
\text { mization with Lower Bound }\end{array}$ & $(5)$ \\
SPAPR-Min & $\begin{array}{l}\text { Spatial Peak-to-Average Power } \\
\text { Ratio Minimization }\end{array}$ \\
PPM & Peak Power Minimization & $(12)$ \\
SPM & Sum Power Minimization & $(27)$ \\
\hline
\end{tabular}

\section{Comparative Performance Analysis}

Hereafter, some comparative simulation results are discussed for the proposed techniques, i.e. WPPMLB and SPAPR-Min. Unless specified otherwise, the number of transmit antennas is fixed to 10, allowing additional degrees of freedom in the optimization problems to be exploited, and the imposed spatial dynamic range in the WPPMLB approach is fixed to $1 \mathrm{~dB}$ (i.e., $\alpha=-1 \mathrm{~dB})^{10}$.

In the context of symbol-level precoding, we consider as benchmarks the sum power minimization (SPM) scheme [9], [10] and the peak power minimization (PPM) scheme [19], [20]. For the sake of completeness, we provide in the following a formulation for the SPM and the PPM optimization problems, respectively:

$$
\begin{aligned}
& \boldsymbol{x}(\boldsymbol{d}, \boldsymbol{H}, \boldsymbol{\gamma})= \arg \min _{\boldsymbol{x}} \quad\|\boldsymbol{x}\|^{2} \\
& \text { s.t. } \quad \mathcal{C} 1:\left|\boldsymbol{h}_{j} \boldsymbol{x}\right|^{2} \unrhd \kappa_{j}^{2} \gamma_{j} \sigma_{z}^{2}, j=1, \ldots, K, \\
& \mathcal{C} 2: \angle \boldsymbol{h}_{j} \boldsymbol{x}=\angle d_{j}, j=1, \ldots, K, \\
& \boldsymbol{x}(\boldsymbol{d}, \boldsymbol{H}, \gamma)=\arg \min _{\boldsymbol{x}} \max _{i=1, \ldots, N_{t}}\left\{\left|x_{i}\right|^{2}\right\} \\
& \text { s.t. } \quad \mathcal{C} 1: \quad\left|\boldsymbol{h}_{j} \boldsymbol{x}\right|^{2} \unrhd \kappa_{j}^{2} \gamma_{j} \sigma_{z}^{2}, j=1, \ldots, K, \\
& \mathcal{C} 2: \quad \angle \boldsymbol{h}_{j} \boldsymbol{x}=\angle d_{j}, j=1, \ldots, K .
\end{aligned}
$$

For the sake of clarity, all the considered symbol-level precoding schemes are summarized in Table II, with their acronyms and the reference to the respective optimization problems.

First of all, we discuss the achieved performance in terms of spatial dynamic range and SPAPR, which are summarized in Table III. The displayed values show a substantial gain of the proposed schemes with respect to the benchmarks, especially in terms of spatial dynamic range, which turns out extremely high when the SPM scheme is used. As already observed, the SPAPR-Min approach is able to reach the lower bound for the spatial dynamic range and the SPAPR. It should be highlighted that this is also possible with the WPPMLB approach by properly setting $\alpha$ to $0 \mathrm{~dB}$, which would come of course with a higher transmit power required, according to the trade-off shown in Fig. 6. Actually, the strength of the WPPMLB approach lies in its flexibility which allows to cope with systems having different requirements, realizing a tradeoff between imposed spatial dynamic range and consumed power.

\footnotetext{
${ }^{10}$ However, it may be set to any value depending on the specific non-linear scenario at hand.
}

Table III: Comparison of the spatial dynamic range and SPAPR values for the different schemes.

\begin{tabular}{ccccc}
\hline & SPAPR-Min & WPPMLB & PPM & SPM \\
\hline Spatial Dynamic Range [dB] & 0 & 1 & 5.1 & 14.4 \\
SPAPR [dB] & 0 & 0.2 & 0.6 & 4.7 \\
\hline
\end{tabular}

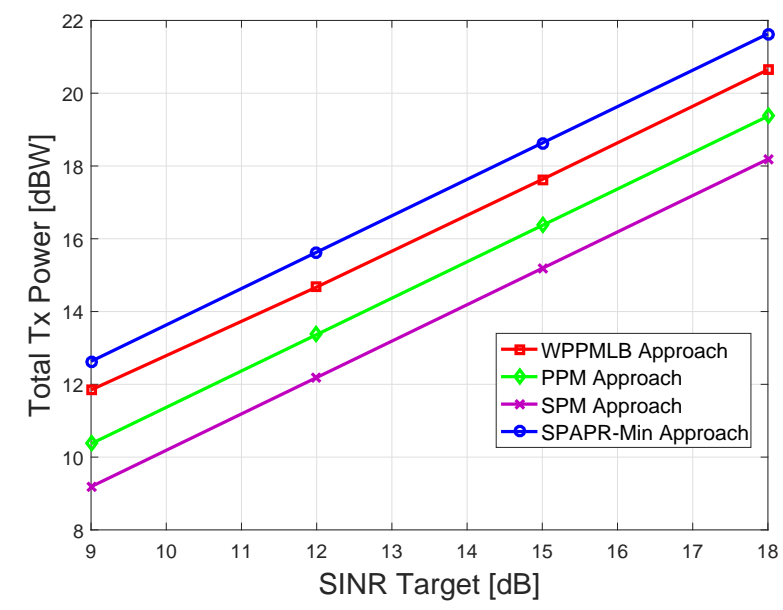

Figure 8: Total transmit power, in $\mathrm{dBW}$, versus target SINR, in $\mathrm{dB}$.

In Fig. 8 the total transmit power, in $\mathrm{dBW}$, is shown as a function of the target SINR, in $\mathrm{dB}$, for the considered techniques. As expectable, the power requirements of the proposed schemes are higher with respect to the benchmarks. In particular, the SPAPR-Min scheme requires a higher total power than the WPPMLB one. Moreover, Fig. 9 displays the average power transmitted by each antenna for the different schemes at hand, for a fixed channel realization. This result allows to better visualize the trade-off of the proposed precoding approaches, which produce a more uniform power distribution amongst the antennas at the expense of a higher consumed power. It shall be highlighted how the more uniform power distribution attained by the proposed approaches implies better performance with respect to the benchmarks (SPM and PPM), over a generic non-linear channel. This is clearly quantified in SER analysis presented later on in this section.

In order to give further insights on the proposed symbollevel precoding schemes, we also consider herein a comparison with the constant envelope precoding of [21]. By construction, this approach designs waveforms with $0 \mathrm{~dB}$ dynamic range (both in space and in time), but it does not achieve the constructive interference effect of the symbol-level schemes at hand. This can be seen in Fig. 10, where the achieved average SINR at the users, in $\mathrm{dB}$, is compared between the different approaches, as a function of the total transmit power in $\mathrm{dBW}$. In fact, we note that an increase in the transmit power, which in turn implies an increase in the interference level, enhances the constructive interference effect in the symbol-level schemes, and results in a considerable gain in the attained SINR. On the other hand, in the scheme of [21] the interference is harmful, thus the SINR has a slower growth when the transmit power increases. In particular, in the case of [21] we 


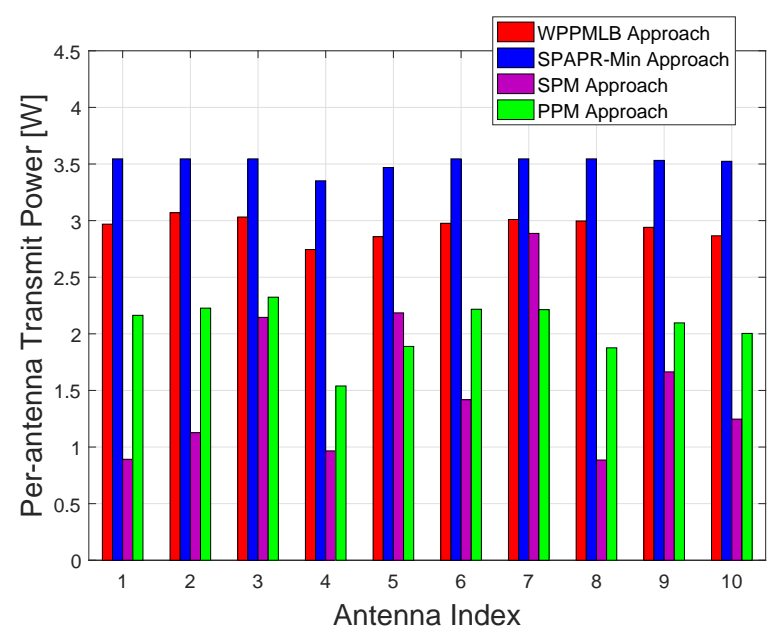

Figure 9: Per-antenna average power, in W, for a fixed channel realization.

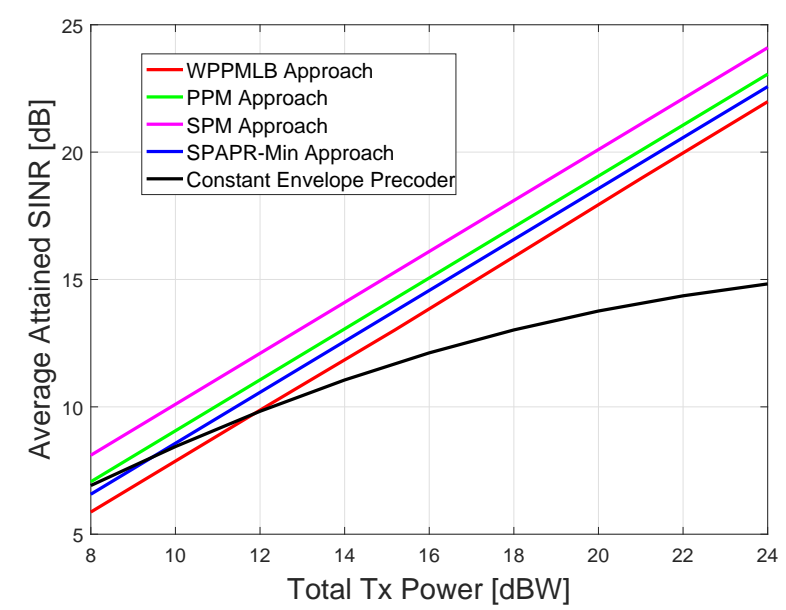

Figure 10: Average achieved SINR, in $\mathrm{dB}$, versus total transmit power, in $\mathrm{dBW}$.

observe a saturation effect of the SINR curve for high transmit power, which results in a maximum achievable SINR. In the remainder of this section, the comparison with the scheme of [21] is also presented in term of SER over a non-linear channel.

In Figs. 11-12 we present a comparative result in terms of SER achieved at the users' side when the schemes at hand are applied over a channel corrupted by non-linearities (besides the MUI and the AWGN), considering a case with 8 transmit antennas and a case with 10 transmit antennas, respectively. This analysis allows to evaluate the overall effectiveness of the proposed techniques with respect to the differential phase shift effect. The results have been obtained by simulating $S=3000$ symbol slots and considering, for both cases, a fixed realization for the channel matrix and a target SINR of $12 \mathrm{~dB}^{11}$. The non-linear model of Fig. 2 has been considered for the

\footnotetext{
${ }^{11}$ This target SINR is also considered for the approach of [21], although in this case it is not always guaranteed that this threshold is achieved, because of the saturation effect of the SINR with the increasing power.
}

simulations ${ }^{12}$. In order to apply the non-linear characteristics, the transmitted waveforms have been modeled by oversampled discrete sequences, by applying a pulse shaping operation to the generated symbols, for each antenna. The pulse shaping operation is performed using a unit energy symmetric pulse waveform $g(t)$. Denoting by $T$ the symbol period and by $\xi$ the oversampling factor, the transmitted waveform for the generic $i$-th antenna can be represented through its discrete samples spaced by $t_{s}=\frac{T}{\xi}$, as follows:

$$
x_{i}^{\mathrm{ovs}}\left[l t_{s}\right]=\sum_{n=0}^{S-1} x_{i}[n] g\left[l t_{s}-n T\right], \quad l=0, \ldots, \xi S-1,
$$

where $n$ indexes the $S$ symbols while $l$ indexes the samples. At the generic $j$-th user, in order to obtain the received signal in the symbol domain, a matched filtering and downsampling operation is applied to the oversampled received waveform $y_{j}^{\text {ovs }}\left[l t_{s}\right]$, as follows:

$$
y_{j}[n]=\sum_{l=0}^{\xi S-1} y_{j}^{\text {ovs }}\left[l t_{s}\right] g\left[l t_{s}-n T\right], \quad n=0, \ldots, S-1 .
$$

The pulses $g(t)$ considered for the simulations are square-rootraised-cosine (SRRC) with a roll-off factor of 0.25 , and the oversampling factor $\xi$ has been set to 10 .

The figures show the obtained SER as a function of the input back-off (IBO), in $\mathrm{dB}$, applied to the signal feeding the non-linear amplifiers. The shape of the obtained curves can be explained by considering that, in general, rescaling the average power of the transmit signals (i.e., applying a back-off with respect to the saturation point of the amplifiers) allows to mitigate the non-linear effects, and therefore to improve the SER performance. On the other hand, the back-off operation reduces the SINR, hence a very high IBO tends to increase the achieved SER. Ultimately there is a trade-off, and the optimal IBO can be identified as the one minimizing the SER.

In both the cases with 8 and 10 antennas, it is visible how the proposed approaches allow to achieve an improved SER with respect to the symbol-level precoding benchmarks, i.e., the PPM approach and the SPM approach ${ }^{13}$. Furthermore, it can be noted that, in most of the simulated cases, the proposed techniques allow the non-linear amplifiers to operate with a lower IBO in the optimal operating point. Interestingly, it turns out that the SPAPR-Min scheme slightly outperforms the WPPMLB one, even when a spatial dynamic range of 0 $\mathrm{dB}$ is imposed in the latter. It is conjectured that this is due to a better constructive interference effect taking place with the

\footnotetext{
${ }^{12}$ It should be mentioned that the absolute average phase rotation, given by the PM characteristic of the amplifiers, is assumed estimated and compensated at the receiver side, based on pilot symbols. This phase recovery and compensation is a necessary operation, without which the detection process cannot proceed, and is normally easy to perform, since it is referred to the average phase shift.

${ }^{13}$ The attained SER values can be reduced by increasing the SINR target. Moreover, although channel coding is out of the scope of this work, it should be noted that a forward error correction (FEC) scheme can strongly boost the overall performance in terms of bit error rate. A study of the proposed schemes including FEC is foreseen in future work.
} 


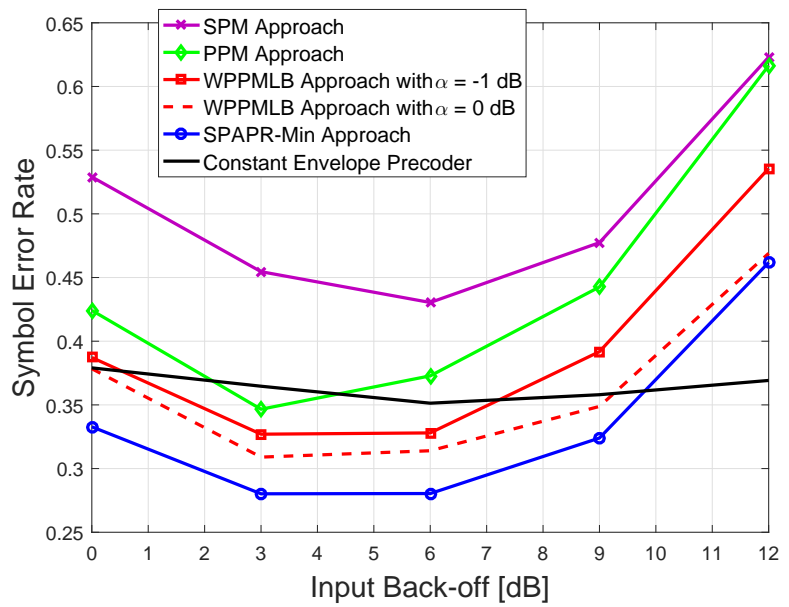

Figure 11: Achieved SER versus IBO, in $\mathrm{dB}$, for a channel with 5 users and 8 antennas.

SPAPR-Min scheme, or even to an improved dynamic in the temporal dimension experienced in such scheme.

The comparison of the proposed schemes with the constant envelope precoder of [21] needs a separate discussion. Remarkably, in the case with 8 antennas of Fig. 11, the proposed approaches outperform the constant envelope precoder in terms of achieved SER. As a matter of fact, the numerical analysis has revealed that the maximum SINR achievable by [21] in this scenario is around $9 \mathrm{~dB}$, due to the aforementioned saturation effect of the SINR. Therefore, the constant envelope precoder cannot guarantee the target SINR of $12 \mathrm{~dB}$. However, the symbol level schemes are able to achieve the guaranteed SINR target. As a result, a better SER performance is attained by the proposed schemes. The situation changes in the case with 10 antennas of Fig. 12. In fact, in turns out that in this scenario the constant envelope scheme can guarantee the target SINR of $12 \mathrm{~dB}$, as well as the proposed schemes. As a consequence, the advantage of [21] in terms of dynamic range of the waveforms (it achieves constant envelope waveforms in space and time) dominates, and a better SER performance is obtained. We conclude that the number of antennas is an important factor in the choice of one scheme over the other. Further, the target SINR is also a relevant factor in the choice, since the scheme in [21] presents a saturation effect (as also shown in Fig. 10).

\section{Out-of-band Radiation}

A final remark about the proposed precoding schemes is related to the out-of-band radiation. In fact, it is well known that the non-linear relation (4) widens the spectrum of the amplified waveforms, determining the transmission of power outside the pulse bandwidth. Considering the signal transmitted by one of the antennas when a $3 \mathrm{~dB}$ IBO is applied, Fig. 13 compares the related power spectral density at the output of the non-liner amplifier, for the symbollevel precoding approaches at hand, together with the case of a non-precoded waveform. Interestingly, it emerges how the proposed approaches (WPPM, WPPMLB and SPAPR-

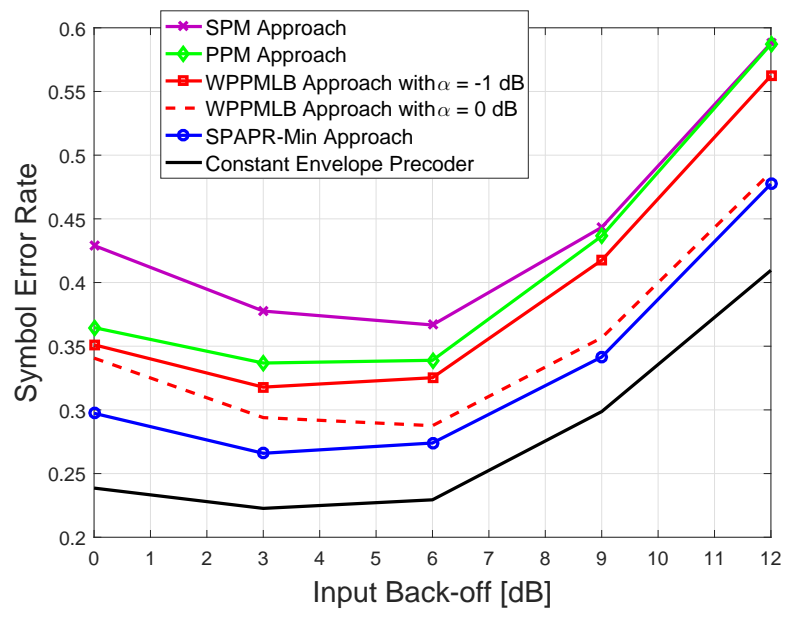

Figure 12: Achieved SER versus IBO, in $\mathrm{dB}$, for a channel with 5 users and 10 antennas.

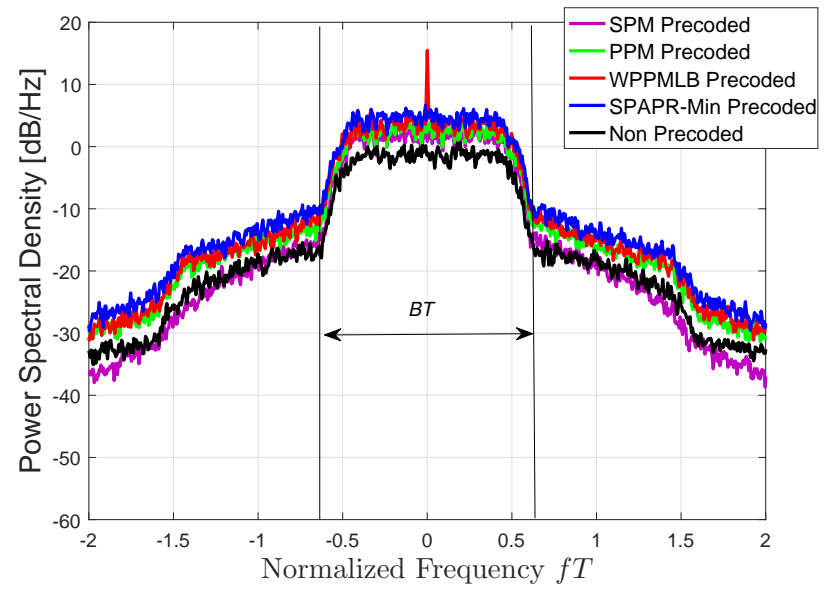

Figure 13: Power spectral density of the output signals with the proposed approaches for a $3 \mathrm{~dB}$ IBO; the normalized frequency $f T$ is considered, with $T$ being the symbol period; the pulse bandwidth is $B T=1.25$.

Min) determine a moderately higher out-of-band radiation with respect to the SPM precoding case and the non-precoded one. This can be explained by considering that the introduced techniques are not improving the signals dynamic properties in the time dimension. Accordingly, a reduction of the out-ofband radiation was out of the scope of this work. Nonetheless, it shall be noted how the relative level of the out-of-band radiation with respect to the in-band radiation for the proposed techniques is comparable with the non-precoded case. Finally, the DC level observable in the WPPMLB case reveals an asymmetric shape for the transmit constellations produced by this approach.

\section{Conclusions}

In the present work, two novel strategies for symbollevel precoding have been proposed, aiming at controlling the per-antenna instantaneous transmit power and at limiting the power imbalances across the different RF chains. A 
first proposed scheme (WPPMLB) performs a weighted perantenna power minimization, imposing a lower bound to the power carried by each transmitted stream. A second scheme (SPAPR-Min) performs the minimization of the spatial peakto-average power ratio. Both the approaches allow to reduce the spatial dynamic of the transmitted waveforms, besides exploiting the constructive interference as in other symbollevel strategies available in the literature. Such feature, which is novel in the context of symbol-level precoding, makes these techniques particularly relevant in systems affected by nonlinear impairments. More specifically, they allow to deal with the problem of differential phase shift, which is characterized in the contribution. The performance of the proposed schemes is assessed through numerical results in terms of spatial dynamic range, spatial peak-to-average power ratio and symbol error rate, in comparison with state of the art symbollevel precoding techniques. The results show how the novel strategies outperform the existent symbol-level approaches with respect to the mentioned metrics. They also motivate using more transmit antennas that served users to improve the waveform characteristics of the transmitted signal. The WPPMLB scheme has been shown more flexible than the SPAPR-Min one, which however is able to achieve a slightly lower symbol error rate. An extension of the proposed schemes aimed at optimizing the transmitted waveforms also in the temporal dimension (besides the spatial one) is foreseen for the future work, so as to cope with frequency selective channels and with the resulting inter-symbol interference.

\section{APPENDIX}

\section{A. Proof of Theorem 1.}

This proof is based on a number of steps through which the optimization problem (5) is transformed into the formulation in (6).

First of all, following the method of [19], [40], [41], the constraint $\mathcal{C} 3$ in (5) can be rewritten, by applying the tangent operator $^{14}$, as:

$$
\frac{\operatorname{Im}\left(\boldsymbol{h}_{j} \boldsymbol{x}\right)}{\operatorname{Re}\left(\boldsymbol{h}_{j} \boldsymbol{x}\right)}=t_{j}, j=1, \ldots, K
$$

where $t_{j}=\tan \left(\angle d_{j}\right)$. However, since the tangent is not a oneto-one function, the following constraints should be added, in order to ensure that the received symbol and the intended one lie in the same quadrant:

$$
\begin{aligned}
& \operatorname{Re}\left(d_{j}\right) \operatorname{Re}\left(\boldsymbol{h}_{j} \boldsymbol{x}\right) \geq 0, j=1, \ldots, K, \\
& \operatorname{Im}\left(d_{j}\right) \operatorname{Im}\left(\boldsymbol{h}_{j} \boldsymbol{x}\right) \geq 0, j=1, \ldots, K .
\end{aligned}
$$

Secondly, the QoS constraint $\mathcal{C} 2$ in the problem (5) can be rewritten, again in the same fashion of [19], [40], [41], referring to the amplitude levels of the in-phase and quadrature components of the corresponding symbols, as follows:

\footnotetext{
${ }^{14}$ This does not apply for data symbols laying on the imaginary axis, since the tangent is not defined in such case. Although this case can be easily handled, it is not considered herein, since we can always assume a phase offset preventing this situation.
}

$$
\begin{aligned}
& \left|\operatorname{Re}\left(\boldsymbol{h}_{j} \boldsymbol{x}\right)\right| \unrhd \sigma_{z} \sqrt{\gamma_{j}}\left|\operatorname{Re}\left(d_{j}\right)\right|, j=1, \ldots, K, \\
& \left|\operatorname{Im}\left(\boldsymbol{h}_{j} \boldsymbol{x}\right)\right| \unrhd \sigma_{z} \sqrt{\gamma_{j}}\left|\operatorname{Im}\left(d_{j}\right)\right|, j=1, \ldots, K,
\end{aligned}
$$

where the absolute value is necessary for accounting negative components. By multiplying both the members of the above equations by $\operatorname{Re}\left(d_{j}\right)$ and $\operatorname{Im}\left(d_{j}\right)$ respectively, the above conditions become:

$$
\begin{aligned}
& \operatorname{Re}\left(d_{j}\right) \operatorname{Re}\left(\boldsymbol{h}_{j} \boldsymbol{x}\right) \unrhd \sigma_{z} \sqrt{\gamma_{j}} \operatorname{Re}^{2}\left(d_{j}\right), j=1, \ldots, K, \\
& \operatorname{Im}\left(d_{j}\right) \operatorname{Im}\left(\boldsymbol{h}_{j} \boldsymbol{x}\right) \unrhd \sigma_{z} \sqrt{\gamma_{j}} \operatorname{Im}^{2}\left(d_{j}\right), j=1, \ldots, K .
\end{aligned}
$$

It is worth noticing that the constraints in (33) include the ones shown in (31).

Thus, the problem becomes:

$$
\begin{aligned}
& \boldsymbol{x}(\boldsymbol{d}, \boldsymbol{H}, \boldsymbol{\gamma}, \boldsymbol{p})=\arg \min _{r, \boldsymbol{x}} \quad r \\
& \text { s.t. } \quad \mathcal{C} 1: \quad \alpha r^{2} \leq \frac{\left|x_{i}\right|^{2}}{p_{i}} \leq r^{2}, i=1, \ldots, N_{t}, \\
& \mathcal{C} 2: \quad \operatorname{Re}\left(d_{j}\right) \operatorname{Re}\left(\boldsymbol{h}_{j} \boldsymbol{x}\right) \unrhd \sigma_{z} \sqrt{\gamma_{j}} \operatorname{Re}^{2}\left(d_{j}\right), \\
& j=1, \ldots, K, \\
& \mathcal{C} 3: \quad \operatorname{Im}\left(d_{j}\right) \operatorname{Im}\left(\boldsymbol{h}_{j} \boldsymbol{x}\right) \unrhd \sigma_{z} \sqrt{\gamma_{j}} \operatorname{Im}^{2}\left(d_{j}\right), j=1, \ldots, K, \\
& \mathcal{C} 4: \frac{\operatorname{Im}\left(\boldsymbol{h}_{j} \boldsymbol{x}\right)}{\operatorname{Re}\left(\boldsymbol{h}_{j} \boldsymbol{x}\right)}=t_{j}, j=1, \ldots, K .
\end{aligned}
$$

Ultimately, the problem can be rewritten in a more compact form as:

$$
\begin{aligned}
& \boldsymbol{x}(\boldsymbol{d}, \boldsymbol{H}, \boldsymbol{\gamma}, \boldsymbol{p})=\arg \min _{r, \boldsymbol{x}} r \\
& \text { s.t. } \quad \mathcal{C} 1: \frac{\left|x_{i}\right|^{2}}{p_{i}} \leq r^{2}, i=1, \ldots, N_{t}, \\
& \mathcal{C} 2: \frac{\left|x_{i}\right|^{2}}{p_{i}} \geq \alpha r^{2}, i=1, \ldots, N_{t}, \\
& \mathcal{C} 3: \operatorname{Re}(\boldsymbol{D}) \operatorname{Re}(\boldsymbol{H} \boldsymbol{x}) \unrhd \boldsymbol{\beta}_{\boldsymbol{R}} \\
& \mathcal{C} 4: \operatorname{Im}(\boldsymbol{D}) \operatorname{Im}(\boldsymbol{H} \boldsymbol{x}) \unrhd \boldsymbol{\beta}_{\boldsymbol{I}} \\
& \mathcal{C} 5: \boldsymbol{T} \operatorname{Re}(\boldsymbol{H} \boldsymbol{x})-\operatorname{Im}(\boldsymbol{H} \boldsymbol{x})=\mathbf{0},
\end{aligned}
$$

where $\boldsymbol{D}=\operatorname{diag}(\boldsymbol{d}), \boldsymbol{T}=\operatorname{diag}\left(t_{1}, \ldots, t_{K}\right), \boldsymbol{\beta}_{\boldsymbol{r}}=\sigma_{z} \sqrt{\boldsymbol{\gamma}} \circ$ $\operatorname{Re}(\boldsymbol{d})^{\circ 2}, \boldsymbol{\beta}_{\boldsymbol{i}}=\sigma_{z} \sqrt{\boldsymbol{\gamma}} \circ \operatorname{Im}(\boldsymbol{d})^{\circ 2}$.

A further step for simplifying the problem (35) is to rewrite it in the real domain, in the stacked variable $\tilde{\boldsymbol{x}}=$ $\left[\operatorname{Re}(\boldsymbol{x})^{T}, \operatorname{Im}(\boldsymbol{x})^{T}\right]^{T} \in \mathbb{R}^{2 N_{t} \times 1}$. To this end, the constraints should be modified accordingly.

Regarding the weighted per-antenna transmit power, it is not difficult to see that:

$$
\frac{\left|x_{i}\right|^{2}}{p_{i}}=\left\|\boldsymbol{B}_{i} \tilde{\boldsymbol{x}}\right\|^{2},
$$

where $\boldsymbol{B}_{i} \in \mathbb{R}^{2 \times 2 N_{t}}$ is a matrix used for selecting $\operatorname{Re}\left(x_{i}\right)$ and $\operatorname{Im}\left(x_{i}\right)$ in the stacked vector $\tilde{\boldsymbol{x}}$ and, $\forall i=1, \ldots, N_{t}$, is defined as:

$$
\frac{1}{\sqrt{p_{i}}}\left[\begin{array}{cc}
\boldsymbol{e}_{i} & \mathbf{0}_{N_{t}} \\
\mathbf{0}_{N_{t}} & \boldsymbol{e}_{i}
\end{array}\right]
$$


with $\boldsymbol{e}_{i}$ being a the $i$-th row of an identity matrix with size $N_{t}$, and $\mathbf{0}_{N_{t}}$ being the all zero entries vector in $\mathbb{R}^{1 \times N_{t}}$.

Concerning the QoS constraints, it is convenient to split the vector $\boldsymbol{H} \boldsymbol{x}$ into its real and imaginary parts:

$$
\begin{aligned}
\boldsymbol{H} \boldsymbol{x}= & \operatorname{Re}(\boldsymbol{H}) \operatorname{Re}(\boldsymbol{x})-\operatorname{Im}(\boldsymbol{H}) \operatorname{Im}(\boldsymbol{x})+ \\
& +\iota[\operatorname{Re}(\boldsymbol{H}) \operatorname{Im}(\boldsymbol{x})+\operatorname{Im}(\boldsymbol{H}) \operatorname{Re}(\boldsymbol{x})],
\end{aligned}
$$

which leads straightforwardly to:

$$
\operatorname{Re}(\boldsymbol{H} \boldsymbol{x})=\boldsymbol{H}_{\mathbf{1}} \tilde{\boldsymbol{x}}, \quad \operatorname{Im}(\boldsymbol{H} \boldsymbol{x})=\boldsymbol{H}_{\mathbf{2}} \tilde{\boldsymbol{x}},
$$

where $\boldsymbol{H}_{\mathbf{1}}=[\operatorname{Re}(\boldsymbol{H}),-\operatorname{Im}(\boldsymbol{H})], \boldsymbol{H}_{\mathbf{2}}=[\operatorname{Im}(\boldsymbol{H}), \operatorname{Re}(\boldsymbol{H})]$.

With the above positions, the problem (35) can be expressed as:

$$
\begin{aligned}
\boldsymbol{x}(\boldsymbol{d}, \boldsymbol{H}, \boldsymbol{\gamma}, \boldsymbol{p})=\arg \min _{r, \boldsymbol{x}} \quad r \\
\text { s.t. } \quad \mathcal{C} 1: \quad\left\|\boldsymbol{B}_{i} \tilde{\boldsymbol{x}}\right\| \leq r, i=1, \ldots, N_{t}, \\
\mathcal{C} 2: \quad\left\|\boldsymbol{B}_{i} \tilde{\boldsymbol{x}}\right\|^{2} \geq \alpha r^{2}, i=1, \ldots, N_{t}, \\
\mathcal{C} 3: \quad \operatorname{Re}(\boldsymbol{D}) \boldsymbol{H}_{\mathbf{1}} \tilde{\boldsymbol{x}} \unrhd \boldsymbol{\beta}_{\boldsymbol{r}}, \\
\mathcal{C} 4: \quad \operatorname{Im}(\boldsymbol{D}) \boldsymbol{H}_{\mathbf{2}} \tilde{\boldsymbol{x}} \unrhd \boldsymbol{\beta}_{\boldsymbol{i}}, \\
\mathcal{C} 5: \quad\left(\boldsymbol{T} \boldsymbol{H}_{\mathbf{1}}-\boldsymbol{H}_{\mathbf{2}}\right) \tilde{\boldsymbol{x}}=\mathbf{0} .
\end{aligned}
$$

Finally, by defining the matrices $\boldsymbol{A}_{i}=-\boldsymbol{B}_{i}^{\dagger} \boldsymbol{B}_{i}$ the problem becomes the one in (6), hence the Theorem 1 is proved.

\section{B. Proof of Theorem 2.}

Retracing the procedure followed in the proof in Appendix $\mathrm{A}$, and specifically considering the equations (30)-(33), the problem (12) can be rewritten as follows:

$$
\begin{aligned}
& \boldsymbol{x}(\boldsymbol{d}, \boldsymbol{H}, \boldsymbol{\gamma})= \arg \min _{\boldsymbol{x}} \frac{\|\boldsymbol{x}\|_{\infty}^{2}}{\|\boldsymbol{x}\|^{2}} \\
& \text { s.t. } \quad \mathcal{C} 1: \quad \operatorname{Re}\left(d_{j}\right) \operatorname{Re}\left(\boldsymbol{h}_{j} \boldsymbol{x}\right) \unrhd \sigma_{z} \sqrt{\gamma_{j}} \operatorname{Re}^{2}\left(d_{j}\right), \\
& j=1, \ldots, K, \\
& \mathcal{C} 2: \quad \operatorname{Im}\left(d_{j}\right) \operatorname{Im}\left(\boldsymbol{h}_{j} \boldsymbol{x}\right) \unrhd \sigma_{z} \sqrt{\gamma_{j}} \operatorname{Im}^{2}\left(d_{j}\right), \\
& j=1, \ldots, K . \\
& \mathcal{C} 3: \quad t_{j} \operatorname{Re}\left(\boldsymbol{h}_{j} \boldsymbol{x}\right)-\operatorname{Im}\left(\boldsymbol{h}_{j} \boldsymbol{x}\right)=0, j=1, \ldots, K .
\end{aligned}
$$

By applying the following equalities:

$$
\begin{aligned}
& \operatorname{Re}\left(\boldsymbol{h}_{j} \boldsymbol{x}\right)=\frac{\boldsymbol{h}_{j} \boldsymbol{x}+\boldsymbol{x}^{\dagger} \boldsymbol{h}_{j}^{\dagger}}{2}, \\
& \operatorname{Im}\left(\boldsymbol{h}_{j} \boldsymbol{x}\right)=\frac{\boldsymbol{h}_{j} \boldsymbol{x}-\boldsymbol{x}^{\dagger} \boldsymbol{h}_{j}^{\dagger}}{2 \iota},
\end{aligned}
$$

the problem (12) can be straightforwardly expressed as in (13). Thus, the Theorem 2 is proved.

\section{REFERENCES}

[1] R. Roy and B. Ottersten, "Spatial division multiple access wireless communication systems," May 1996, US Patent 5,515,378. [Online]. Available: https://www.google.com/patents/US5515378

[2] Y.-F. Liu, Y.-H. Dai, and Z.-Q. Luo, "Coordinated beamforming for MISO interference channel: Complexity analysis and efficient algorithms," IEEE Transactions on Signal Processing, vol. 59, no. 3, pp. 1142-1157, 2011.

[3] M. Bengtsson and B. Ottersten, "Optimal and suboptimal transmit beamforming," in Handbook of Antennas in Wireless Communications. CRC Press, 2001.

[4] M. Schubert and H. Boche, "Solution of the multiuser downlink beamforming problem with individual SINR constraints," IEEE Transactions on Vehicular Technology, vol. 53, no. 1, pp. 18-28, Jan 2004.

[5] W. Yu and T. Lan, "Transmitter optimization for the multi-antenna downlink with per-antenna power constraints," IEEE Transactions on Signal Processing, vol. 55, no. 6, pp. 2646-2660, June 2007.

[6] G. Dartmann, X. Gong, W. Afzal, and G. Ascheid, "On the duality of the max min beamforming problem with per-antenna and per-antennaarray power constraints," IEEE Transactions on Vehicular Technology, vol. 62, no. 2, pp. 606-619, Feb 2013.

[7] C. Masouros and E. Alsusa, "Dynamic linear precoding for the exploitation of known interference in MIMO broadcast systems," IEEE Transactions on Wireless Communications, vol. 8, no. 3, pp. 1396-1404, March 2009.

[8] C. Masouros, "Correlation rotation linear precoding for MIMO broadcast communications," IEEE Transactions on Signal Processing, vol. 59, no. 1, pp. 252-262, Jan. 2011.

[9] M. Alodeh, S. Chatzinotas, and B. Ottersten, "Constructive multiuser interference in symbol level precoding for the MISO downlink channel," IEEE Transactions on Signal Processing, vol. 63, no. 9, pp. 2239-2252, May 2015.

[10] M. Alodeh, S. Chatzinotas, and B. Ottersten, "Symbol-level multiuser MISO precoding for multi-level adaptive modulation," IEEE Transactions on Wireless Communications, vol. 16, no. 8, pp. 5511-5524, Aug 2017.

[11] M. Alodeh, S. Chatzinotas, and B. Ottersten, "Method and device for symbol-level multiuser precoding," Patent PCT/EP2016/072 600, 2016.

[12] M. Alodeh, S. Chatzinotas, and B. Ottersten, "Energy-efficient symbollevel precoding in multiuser MISO based on relaxed detection region," IEEE Transactions on Wireless Communications, vol. 15, no. 5, pp. 3755-3767, May 2016.

[13] C. Masouros and G. Zheng, "Exploiting known interference as green signal power for downlink beamforming optimization," IEEE Transactions on Signal Processing, vol. 63, no. 14, pp. 3628-3640, July 2015.

[14] G. Auer, V. Giannini, C. Desset, I. Gódor, P. Skillermark, M. Olsson, M. A. Imran, D. Sabella, M. J. Gonzalez, O. Blume, and A. Fehske, "How much energy is needed to run a wireless network?" IEEE Wireless Communications, vol. 18, no. 5, pp. 40-49, October 2011.

[15] S. Cripps, RF Power Amplifiers for Wireless Communications, 2nd ed. Artech House Microwave Library, 2006.

[16] R. Piazza, M. R. B. Shankar, and B. Ottersten, "Data predistortion for multicarrier satellite channels based on direct learning," IEEE Transactions on Signal Processing, vol. 62, no. 22, pp. 5868-5880, Nov 2014.

[17] D. Spano, D. Christopoulos, S. Andrenacci, S. Chatzinotas, J. Krause, and B. Ottersten, "Total degradation analysis of precoded signals onto non-linear satellite channels," in 21st Ka and Broadband Communications Conference, Oct 2015.

[18] D. Spano, M. Alodeh, S. Chatzinotas, J. Krause, and B. Ottersten, "Spatial PAPR reduction in symbol-level precoding for the multi-beam satellite downlink," in 2017 IEEE 18th International Workshop on Signal Processing Advances in Wireless Communications (SPAWC), July 2017. [Online]. Available: http://hdl.handle.net/10993/31224

[19] D. Spano, M. Alodeh, S. Chatzinotas, and B. Ottersten, "Per-antenna power minimization in symbol-level precoding," in 2016 IEEE Global Communications Conference (GLOBECOM), Dec 2016, pp. 1-6.

[20] M. Alodeh, D. Spano, S. Chatzinotas, and B. Ottersten, "Peak power minimization in symbol-level precoding for cognitive miso downlink channels," in 2016 IEEE International Conference on Digital Signal Processing (DSP), Oct 2016, pp. 240-244.

[21] S. K. Mohammed and E. G. Larsson, "Per-antenna constant envelope precoding for large multi-user MIMO systems," IEEE Transactions on Communications, vol. 61, no. 3, pp. 1059-1071, March 2013. 
[22] C. Studer and E. G. Larsson, "PAR-aware large-scale multi-user MIMOOFDM downlink," IEEE Journal on Selected Areas in Communications, vol. 31, no. 2, pp. 303-313, February 2013.

[23] C. Mollén and E. G. Larsson, "Multiuser MIMO precoding with perantenna continuous-time constant-envelope constraints," in 2015 IEEE 16 th International Workshop on Signal Processing Advances in Wireless Communications (SPAWC), June 2015, pp. 261-265.

[24] M. Alodeh, D. Spano, S. Chatzinotas, and B. Ottersten, "Faster-thanNyquist spatiotemporal symbol-level precoding in the downlink of multiuser MISO channels," in 2017 IEEE International Conference on Acoustics, Speech and Signal Processing (ICASSP), March 2017.

[25] E. Casini, R. D. Gaudenzi, and A. Ginesi, "DVB-S2 modem algorithms design and performance over typical satellite channels," International Journal of Sat. Comm. and Netw., vol. 22, no. 3, pp. 281-318, 2004. [Online]. Available: http://dx.doi.org/10.1002/sat.791

[26] D. Spano, S. Chatzinotas, J. Krause, and B. Ottersten, "Symbollevel precoding with per-antenna power constraints for the multi-beam satellite downlink," in 2016 8th Advanced Satellite Multimedia Systems Conference and the 14th Signal Processing for Space Communications Workshop (ASMS/SPSC), Sept 2016, pp. 1-8.

[27] ETSI EN 302 307-1, "Digital video broadcasting (DVB); second generation framing structure, channel coding and modulation systems for broadcasting, interactive services, news gathering and other broadband satellite applications; part 1: DVB-S2."

[28] ETSI EN 302 307-2, "Digital video broadcasting (DVB); second generation framing structure, channel coding and modulation systems for broadcasting, interactive services, news gathering and other broadband satellite applications; part 2: DVB-S2 extensions (DVB-S2X)."

[29] A. A. M. Saleh, "Frequency-independent and frequency-dependent nonlinear models of TWT amplifiers," IEEE Transactions on Communications, vol. 29, no. 11, pp. 1715-1720, November 1981.

[30] M. O'Droma, S. Meza, and Y. Lei, "New modified Saleh models for memoryless nonlinear power amplifier behavioural modelling," IEEE Communications Letters, vol. 13, no. 12, pp. 1007-1007, December 2009.

[31] C. Mollén, E. G. Larsson, and T. Eriksson, "Waveforms for the massive MIMO downlink: Amplifier efficiency, distortion, and performance," IEEE Transactions on Communications, vol. 64, no. 12, pp. 5050-5063, Dec 2016.

[32] A. Beck, A. Ben-Tal, and L. Tetruashvili, "A sequential parametric convex approximation method with applications to nonconvex truss topology design problems," Journal of Global Optimization, vol. 47, no. 01 , pp. 29-51, 2010.

[33] O. Mehanna, K. Huang, B. Gopalakrishnan, A. Konar, and N. D. Sidiropoulos, "Feasible point pursuit and successive approximation of non-convex QCQPs," IEEE Signal Processing Letters, vol. 22, no. 7, pp. 804-808, July 2015.

[34] M. Hong, Q. Li, and Y. F. Liu, "Decomposition by successive convex approximation: A unifying approach for linear transceiver design in heterogeneous networks," IEEE Transactions on Wireless Communications, vol. 15, no. 2, pp. 1377-1392, Feb 2016.

[35] I. Iofedov and D. Wulich, "MIMO-OFDM with nonlinear power amplifiers," IEEE Transactions on Communications, vol. 63, no. 12, pp. 4894-4904, Dec 2015.

[36] Y. Rahmatallah and S. Mohan, "Peak-to-average power ratio reduction in OFDM systems: A survey and taxonomy," IEEE Communications Surveys Tutorials, vol. 15, no. 4, pp. 1567-1592, 2013.

[37] W. Dinkelbach, "On nonlinear fractional programming," Management Science, vol. 13, no. 7, pp. 492-498, March 1967.

[38] R. Jagannathan, "On some properties of programming problems in parametric form pertaining to fractional programming," Management Science, vol. 12, no. 7, pp. 609-615, 1966.

[39] A. Hjørungnes, Complex-Valued Matrix Derivatives With Applications in Signal Processing and Communications. Cambridge University Press, 2011.

[40] A. Kalantari, M. Soltanalian, S. Maleki, S. Chatzinotas, and B. Ottersten, "Secure M-PSK communication via directional modulation," in 2016 IEEE International Conference on Acoustics, Speech and Signal Processing (ICASSP), March 2016, pp. 3481-3485.

[41] A. Kalantari, M. Soltanalian, S. Maleki, S. Chatzinotas, and B. Ottersten, "Directional modulation via symbol-level precoding: A way to enhance security," IEEE Journal of Selected Topics in Signal Processing, vol. 10, no. 8, pp. 1478-1493, Dec 2016.

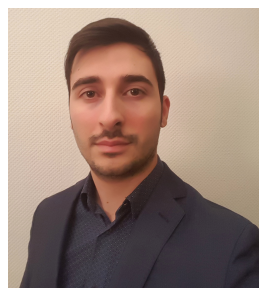

Danilo Spano (S'14) was born in Lecce, Italy, in 1989. He received the B.Sc. degree (cum laude) in Information Engineering and the M.Sc. degree (cum laude) in Telecommunications Engineering from the University of Salento, Lecce, Italy, in 2012 and 2014, respectively.

$\mathrm{He}$ is currently pursuing the Ph.D. degree in electrical engineering at the Interdisciplinary Centre for Security, Reliability, and Trust, University of Luxembourg, Luxembourg. His research interests include signal processing for multiuser wireless communications, satellite communications, interference management, and wireless localization. Mr. Spano was awarded in 2015 with the AFR scholarship from Luxembourg National Research Fund, for the study of interference management techniques in multi-antenna systems.

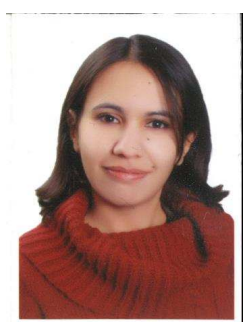

Maha Alodeh (S'11-M'16) received the bachelor's degree in electrical engineering from the University of Jordan, Amman, Jordan, in 2010, and the Ph.D. degree in electrical engineering from the Interdisciplinary Centre for Security, Reliability, and Trust, University of Luxembourg, Luxembourg, in 2015.

She held research positions in University of Jordan, Eurecom-Sophia Antipolis, SnT-University of Luxembourg. She was awarded with AFR scholarship from Luxembourg National Research Fund to carry her $\mathrm{PhD}$ studies. Her research interests include signal processing for wireless and satellite communications with focus on interference management and cognitive radios. She is currently a postdoctoral research associate in SIGCOM group, SnT-University of Luxembourg.

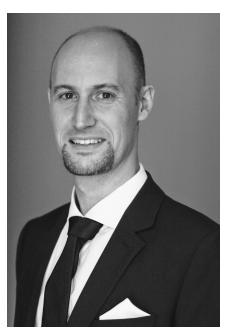

Symeon Chatzinotas (S'06-M'09-SM'13) received the M.Eng. degree in telecommunications from the Aristotle University of Thessaloniki, Thessaloniki, Greece, in 2003, and the M.Sc. and Ph.D. degrees in electronic engineering from the University of Surrey, Surrey, U.K., in 2006 and 2009, respectively.

$\mathrm{He}$ was involved in numerous Research and Development projects for the Institute of Informatics Telecommunications, National Center for Scientific Research Demokritos, the Institute of Telematics and Informatics, Center of Research and Technology Hellas, and the Mobile Communications Research Group, Center of Communication Systems Research, University of Surrey. He is currently the Deputy Head of the SIGCOM Research Group, Interdisciplinary Centre for Security, Reliability, and Trust, University of Luxembourg, Luxembourg and Visiting Professor at the University of Parma, Italy. He has over 250 publications, 2000 citations, and an H-Index of 24 according to Google Scholar. His research interests include multiuser information theory, co-operative/cognitive communications, and wireless networks optimization. He was a co-recipient of the 2014 Distinguished Contributions to Satellite Communications Award, and the Satellite and Space Communications Technical Committee, the IEEE Communications Society, and the CROWNCOM 2015 Best Paper Award. 


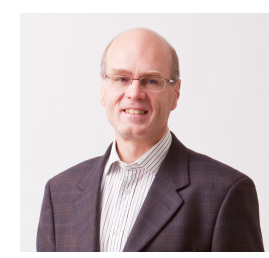

Björn Ottersten (S'87-M'89-SM'99-F'04) was born in Stockholm, Sweden, in 1961. He received the M.S. degree in electrical engineering and applied physics from Linköping University, Linköping, Sweden, in 1986, and the Ph.D. degree in electrical engineering from Stanford University, Stanford, CA, USA, in 1990.

He has held research positions with the Department of Electrical Engineering, Linköping University, the Information Systems Laboratory, Stanford University, the Katholieke Universiteit Leuven, Leuven, Belgium, and the University of Luxembourg, Luxembourg. From 1996 to 1997, he was the Director of Research with ArrayComm, Inc., a startup in San Jose, CA, USA, based on his patented technology. In 1991, he was appointed a Professor of signal processing with the Royal Institute of Technology (KTH), Stockholm, Sweden. From 1992 to 2004, he was the Head of the Department for Signals, Sensors, and Systems, KTH, and from 2004 to 2008, he was the Dean of the School of Electrical Engineering, KTH. $\mathrm{He}$ is currently the Director for the Interdisciplinary Centre for Security, Reliability and Trust, University of Luxembourg. As Digital Champion of Luxembourg, he acts as an Adviser to the European Commission. He was a recipient of the IEEE Signal Processing Society Technical Achievement Award in 2011 and the European Research Council advanced research grant twice, in 2009-2013 and in 2017-2022. He has co-authored journal papers that received the IEEE Signal Processing Society Best Paper Award in 1993, 2001, 2006, and 2013, and seven other IEEE conference papers best paper awards. $\mathrm{He}$ has served as an Associate Editor for the IEEE TRANSACTIONS ON SIGNAL PROCESSING and the Editorial Board of the IEEE Signal Processing Magazine. He is currently the Editor-in-Chief of EURASIP Signal Processing Journal and a member of the editorial boards of EURASIP Journal of Applied Signal Processing and Foundations and Trends of Signal Processing. He is a fellow of EURASIP. 